\title{
Microstructural and defect analysis of metal nanoparticles in functional catalysts by diffraction and electron microscopy: The $\mathrm{Cu} / \mathrm{ZnO}$ catalyst for methanol synthesis
}

\author{
Timur Kandemir ${ }^{a}$, Igor Kasatkin ${ }^{a}$, Frank Girgsdies ${ }^{a}$, Stefan Zander ${ }^{a}$, Stefanie Kühl ${ }^{a}$, Michael Tovar ${ }^{b}$, Robert \\ Schlögl ${ }^{* a}$, Malte Behrens*a \\ ${ }^{a}$ Fritz-Haber-Institut der Max-Planck-Gesellschaft, Department of Inorganic Chemistry, Faradayweg 4-6, \\ 14195 Berlin, Germany \\ ${ }^{\mathrm{b}}$ Helmholtz-Zentrum Berlin, Hahn-Meitner-Platz 1, 14109 Berlin, Germany
}

\begin{abstract}
The application of different methods for a microstructural analysis of functional catalysts is reported for the example of different $\mathrm{Cu} / \mathrm{ZnO}$-based methanol synthesis catalysts. Transmission electron microscopy and diffraction were used as complementary techniques to extract information on the size and the defect concentration of the $\mathrm{Cu}$ nano-crystallites. The results, strengths and limitations of the two techniques and of different evaluation methods for line profile analysis of diffraction data including Rietveld-refinement, Scherrer- and (modified) WilliamsonHall-analyses, single peak deconvolution and whole powder pattern modeling are compared and critically discussed. It was found that in comparison with a macrocrystalline pure Cu sample, the catalysts were not only characterized by a smaller crystallite size, but also by a high concentration of lattice defects, in particular stacking faults. Neutron diffraction was introduced as a valuable tool for such analysis, because of the larger number of higher-order diffraction peaks that can be detected with this method. An attempt is reported to quantify the different types of defects for a selected catalyst.
\end{abstract}

Keywords: Microstructure, Defects, Diffraction, Electron Microscopy, Copper, Methanol Synthesis

\section{Introduction}

The term "microstructure" summarizes all structural features of a solid state material beyond the ideal infinitely extended crystal structure, which in an analogous notation may be termed "picostructure" as it comprises the bond angles and distances between atoms on a picometerscale and their periodicity. The microstructure includes particle size and shape, which can be studied conveniently with an optical or electron microscope in the micro- and nanometer range, respectively, but also other deviations from the ideal crystal lattice such as point, line and planar defects and clusters thereof as well as grain boundaries, interfaces, and crystal mosaicity. In the field of heterogeneous catalysis, knowledge of the microstructure of catalytically active phases is one important goal of catalyst characterization. This is not only true for the particle size as one of the determining factors for the specific accessible active surface area, but in particular also for the intrinsic properties of the surface and its faceting, which is determined by particle shape and size and surface defects. Such steps and kinks can substantially contribute to the catalytic performance in structure sensitive reactions. The increasing understanding of these effects was greatly fertilized by DFT calculations that showed, e.g., how lattice strain, surface steps and kinks or their poisoning will affect catalytic reactions such as ammonia synthesis [1], syngas chemistry [2-3] or CO oxidation [4]. 
In experimental approaches, scanning probe microscopy and related techniques are very suitable tools to study the microstructure of model catalysts comprised of nanoparticles grown on flat surfaces [5]. In case of technically applied catalysts, however, usually powder diffraction methods and electron microscopy are used as complementary integral and local methods. While the latter allows a more or less direct observation of microstructural features [6], the former requires careful evaluation and analysis of the diffraction pattern and peak profiles [7]. In catalysis the most popular way of such evaluation is the application of the Scherrer formula on the width of a selected XRD peak. There are some examples of a deeper analysis of the microstructure of powder catalysts in the literature, e.g. for $\mathrm{Au} / \mathrm{ZrO}{ }_{2}$ [8], $\mathrm{Pt} / \mathrm{SiO}_{2}[9], \mathrm{Ag} / \mathrm{Al}_{2} \mathrm{O}_{3}[10]$ and $\mathrm{Ni}$-based hydrogenation catalysts [11]. However, often the routine microstructural analysis of diffraction data does not go beyond the simple estimation of the volume-weighted average crystallite domain size, which is obtained as the result of the Scherrer formula. One limitations of this analysis, which is unfortunately often not considered, is that it a priori assumes the absence of other peak broadening effects like lattice strain. Furthermore, it is usually only applied on one single peak, typically the strongest non-overlapping XRD line, and in such case neglects any anisotropic peak broadening effects. It also only yields average sizes and not size distributions, and - if performed on a single peak - is only valid for the crystallite dimension normal to the diffracting planes of the peak under study. Other complementary and more sophisticated methods for a microstructural analysis of diffraction data are available to address these issues [12-14]. These methods have mostly been developed in the field of materials science, e.g for metallurgy of construction materials, but can in principle also be applied for catalysts.

Two characteristics of applied catalysts need special attention and often complicate a straightforward adoption of these concepts. These are that i.) catalysts usually are multi-phase composites, comprised for instance of active phase and support, which generate overlapping patterns while typically a special interest exists in only one, often in the minority phase; ii) highly active catalysts typically are nano-structured and often only weakly diffracting materials $[7,15]$. In the recent past, our group has worked extensively on preparation and characterization of a family of catalysts that is to a lower extent affected by these problems, while still representing (or being closely related to) industrially relevant catalyst materials: $\mathrm{Cu} / \mathrm{ZnO}$-based catalysts for methanol synthesis. In the industrial formulation, the active $\mathrm{Cu}$ phase is the major component with a content of $50 \%$ or more and the $\mathrm{Cu}$ particle size in these catalysts is at the range of 5-15 $\mathrm{nm}$ [16] thus large enough to yield reasonable diffraction patterns of sufficient quality. Furthermore, it has been shown that the microstructure, in particular Cu lattice strain [17] and defect density [16] of the Cu phase, is an indicator of an increased catalytic performance and, thus, a microstructural analysis is directly relevant for understanding the catalysis of methanol synthesis. In a recent report [18], we have shown that the concentration of planar defects in the bulk of the $\mathrm{Cu}$ nanoparticles scales linearly with the surface area-normalized activity of the catalysts. This observation can be explained by the changes in surface faceting that are introduced by the termination of extended defects at the exposed surface of the particles forming steps and kinks. In collaboration with the SUNCAT group at Stanford, the higher catalytic activity was confirmed by DFT calculations that clearly showed that surfaces steps that were stabilized by planar bulk defects exhibit more favorable adsorption properties compared to flat $\mathrm{Cu}$ surfaces [18].

During this work, a set of characterization data, diffraction of neutrons and $\mathrm{X}$-rays as well as transmission electron microscopy images, has been collected for $\mathrm{Cu} / \mathrm{ZnO} /\left(\mathrm{Al}_{2} \mathrm{O}_{3}\right)$ catalysts with different preparation history. Herein, we report a comparative microstructural analysis of a selection from these high-performance catalysts. The aim of this paper is to develop a consistent picture of the microstructure of $\mathrm{Cu} / \mathrm{ZnO}$-based methanol synthesis catalysts from different characterization techniques (TEM and diffraction) using various evaluation methods, but also to critically assess the different approaches and to review their limitations as well as their strengths in a scholastic manner. Furthermore, we want to advertise the careful application of sophisticated evaluation methods of diffraction data to characterize the microstructure of 
catalysts.

\section{Materials and Methods}

Four different samples were investigated in this study: two differently prepared ternary catalysts consisting of $\mathrm{Cu} / \mathrm{ZnO} / \mathrm{Al}_{2} \mathrm{O}_{3}$ (labeled CZA-1 and CZA-2), one binary $\mathrm{Cu} / \mathrm{ZnO}$ catalyst that does not contain the alumina structural promoter (labeled $\mathrm{CZ}$ ) as well as a macrocrystalline pure Cu-reference (labeled C-ref). All three catalyst samples are active in methanol synthesis and their relative catalytic performance as well as some major characterization data, which in part has been already published previously [18], is compiled in Table 1.

Table 1: Preparation conditions, selected characterization results and relative intrinsic activities in methanol synthesis under industrial conditions of the three catalysts samples $C Z$, CZA-1 and CZA-2 and the pure Cu reference C-ref (results previously published in ref. [18]).

\begin{tabular}{|l|l|l|l|l|l|}
\hline & $\begin{array}{l}\text { Precipitation } \\
\text { conditions } \\
\mathrm{pH} / \mathrm{T}\left({ }^{\circ} \mathrm{C}\right) / \\
\text { agegin }(\mathrm{min})\end{array}$ & $\begin{array}{l}\mathrm{Cu}: \mathrm{Zn}: \mathrm{Al} \\
(\text { nominal })\end{array}$ & $\begin{array}{l}\mathrm{BET} \text { Surface } \\
\mathrm{area}^{1}\left(\mathrm{~m}^{2} \mathrm{~g}^{-1}\right)\end{array}$ & $\begin{array}{l}\mathrm{N}_{2} \mathrm{O} \text { Surface } \\
\operatorname{area}^{2}\left(\mathrm{~m}^{2} \mathrm{~g}^{-1}\right)\end{array}$ & $\begin{array}{l}\text { Intrinsic } \\
\text { activity } \\
\text { normalized })\end{array}$ \\
\hline C-ref & $6.5 / 65 / 180$ & $100: 0: 0$ & 34 & 6 & 0 \\
\hline $\mathrm{CZ}$ & $6.5 / 65 / 75$ & $70: 30: 0$ & 26 & 26 & 37 \\
\hline $\mathrm{CZA}-1$ & $6.5 / 65 /-$ & $63 / 27 / 10$ & 62 & 24 & 29 \\
\hline $\mathrm{CZA}-2$ & $6.5 / 65 / 60$ & $63 / 27 / 10$ & 91 & 30 & 100 \\
\hline
\end{tabular}

of the calcined catalysts; ${ }^{2}$ of the reduced catalyst; ${ }^{3}$ per Cu surface area, measured at $210{ }^{\circ} \mathrm{C}$ and 60 bar $\mathrm{CO} / \mathrm{CO}_{2} / \mathrm{H}_{2}$

\subsection{Sample preperation}

The pure $\mathrm{Cu}$ reference catalyst $\mathrm{C}$-ref was prepared by co-precipitation of a $\mathrm{Cu}^{2+}$ nitrate solution $(1 \mathrm{M})$ with a basic solution of $\mathrm{Na}_{2} \mathrm{CO}_{3}(1.6 \mathrm{M})$ as the precipitating agent. The reaction was carried out at constant $\mathrm{pH} 6.5$ at $65^{\circ} \mathrm{C}$ with an ageing time of $180 \mathrm{~min}$. The green powder was isolated by filtration, washed with water and dried. Upon calcination in air at $330{ }^{\circ} \mathrm{C}$ for $3 \mathrm{~h}$ the hydroxide carbonate precursor was converted into the oxide, $\mathrm{CuO}$. The binary sample $\mathrm{CZ}$ was synthesized accordingly, but using a mixed $\mathrm{Cu}^{2+} / \mathrm{Zn}^{2+}$ nitrate solution (70:30 molar ratio). The ageing time was expanded until $30 \mathrm{~min}$ after a characteristic minimum in $\mathrm{pH}$ and a change in color from blue to bluish-green occurred [19-20]. A ternary benchmark $\mathrm{Cu} / \mathrm{ZnO} / \mathrm{Al}_{2} \mathrm{O}_{3}$ catalyst CZA-2 was prepared accordingly, but with addition of $10 \mathrm{~mol}-\%$ Al while the $\mathrm{Cu}: \mathrm{Zn}$ ratio was maintained $(\mathrm{Cu}: \mathrm{Zn}: \mathrm{Al}=63: 27: 10)$. This catalyst is a laboratory reproduction of the industrial catalyst following the well-established recipe developed by ICl in the 1960s [20-21]. Another ternary catalyst CZA-1 was synthesized from an amorphous unaged precursor which was prepared by co-precipitation of a mixed $\mathrm{Cu}^{2+} / \mathrm{Zn}^{2+}$ nitrate solution (1 $\mathrm{M}, 70: 30$ ) with an aqueous solution of $\mathrm{NaCO}_{3}(1.6 \mathrm{M})$ containing $10 \mathrm{wt} \% \mathrm{Na}_{2} \mathrm{Al}_{2} \mathrm{O}_{4}$. The reaction was carried out at constant $\mathrm{pH} 6.5$ at $65{ }^{\circ} \mathrm{C}$ with simultaneous continuous pumping of the slurry to the spraydryer $(21.6$ $\mathrm{ml} / \mathrm{min}$ ) [22]. The residence time in the precipitation reactor was estimated to ca. $16 \mathrm{~min}$. Thus in comparison with $\mathrm{CZ}$ and $\mathrm{CZA}-2$, this catalyst precursor can be considered as unaged. Afterwards the blue powder was washed, filtered and spray dried again. Upon calcination in air at $330{ }^{\circ} \mathrm{C}$ for $3 \mathrm{~h}$ all three catalyst precursors were converted into a mixture of the oxides. Around $7 \mathrm{~g}$ of a sieve fraction $(250-355 \mu \mathrm{m})$ of the calcined samples were reduced in a fixed bed reactor (TPDRO 1100, CE instruments) in $5 \% \mathrm{H}_{2} / \mathrm{He}$ at $200{ }^{\circ} \mathrm{C}\left(1 \mathrm{~K} \mathrm{~min}{ }^{1}, 80 \mathrm{ml} \mathrm{min}{ }^{1}\right)$ for $13 \mathrm{~h}$ and finally in $100 \% \mathrm{H}_{2}$ at $250{ }^{\circ} \mathrm{C}$ for $5 \mathrm{~h}$. The majority of the sample was used for the neutron diffraction experiments. A smaller part was passivated with $\mathrm{N}_{2} \mathrm{O}$ and used for the catalytic tests reported in Table 1. 


\subsection{Neutron diffraction}

Approximately $5 \mathrm{~cm}^{3}$ of the reduced sieve fractions were transferred under inert conditions into an indium-sealable vanadium sample holder for neutron diffraction. Neutron scattering data were collected using the Fine Resolution Powder Diffractometer (FIREPOD, E9) at BER-II research reactor hosted by Helmholtz Zentrum Berlin (HZB). The instrument works in Debye Scherrer geometry. The area detector consisting of 64 single ${ }^{3} \mathrm{He}$ detectors covers an angular range of $0^{\circ}-165^{\circ} 2 \theta$ with an angular resolution of 0.1 . By applying (711)-reflection plane of the Ge primary monochromator the wavelength $1.3077 \AA$ was chosen for the experiments. By this sufficient quality data could be gained within a scan time of $24 \mathrm{~h}$ per sample.

\subsection{X-ray diffraction $(\mathrm{XRD})$}

The catalyst CZA-1 was additionally investigated with XRD. The calcined materials was reduced before the measurement in an Anton Paar XRK 900 reaction chamber on a Stoe thetatheta diffractometer ( $\mathrm{Cu} \mathrm{K} \alpha$ radiation, $\lambda=1.5418 \AA$ ) equipped with a secondary graphite monochromator and a scintillation counter. For reduction the temperature was increased linearly in a $5 \% \mathrm{H}_{2}$ in He atmosphere $(100 \mathrm{ml} / \mathrm{min}, 2 \mathrm{~K} / \mathrm{min})$ to $250^{\circ} \mathrm{C}$ and maintained isothermal for $2 \mathrm{~h}$. The reduced catalyst was cooled to room temperature in the reduction gas before collection of XRD patterns $\left(30-100^{\circ} 2 \theta, 0.02^{\circ}\right.$ steps, 16 s counting/step).

\subsection{Diffraction data analysis}

For sake of comparability, the diffraction data obtained from the X-ray or neutron diffractometer with different wavelengths $\lambda$ was transformed from solid scattering angle $(2 \theta)$ to a common abscissa in reciprocal space, $Q$, by Bragg's law:

$$
Q=\frac{4 \pi \sin \theta}{\lambda}
$$

\subsubsection{Instrumental broadening}

The measured scattered intensity $\left(I_{\mathrm{Qm}}\right)$ was deconvoluted to account for the physical broadening of the sample due to size and structural imperfections $\left(I_{Q s}\right)$ and the instrumental broadening $\left(I_{Q i}\right)$, caused (in the case of $x$-rays) by wavelength dispersion and slits [23-24] as well as axial beam divergence caused by monochromator geometry (in neutron diffraction) [25]. To determine instrumental resolution reference materials were measured [24]. For the present study $\mathrm{LaB}_{6}$ was used for the $\mathrm{XRD}$ and $\mathrm{Y}_{2} \mathrm{O}_{3}$ for the neutron diffraction measurements. An attempt to describe the $2 \theta$-dependence of the instrumental contribution was done by the experimental function described first by Caglioti et al. [26], later simplified by Rietveld et al. [27]. A detailed description of modeling the instrumental resolution and deconvolution of the instrumental broadening can be found in the supporting information. Before peak deconvolution, the raw data has been smoothed using the Savitzky-Golay filter.

\subsubsection{Analytical profile functions for pattern decomposition}

For the application of pattern decomposition methods, the experimentally observed line profiles in constant wavelength X-ray and neutron diffraction data were described by pseudoVoigt functions (linear combination of Gaussian and Lorentzian profiles) as introduced by Wertheim et al. [28] with a mixing parameter $\eta$ varying in a range from 0 (pure Gaussian) to 1 (pure Lorentzian): 


$$
I_{Q_{m}}=\eta \cdot L(Q)+(1-\eta) \cdot G(Q) \text { with } 0<\eta<1
$$

with

$$
G(Q)=\exp \left[-\ln (2) \cdot\left(\frac{Q-Q_{0}}{H_{G}}\right)^{2}\right] \text { and } L(Q)=\frac{1}{1+\left(\frac{Q-Q_{0}}{H_{L}}\right)^{2}}
$$

The mixing parameter $\eta$ relates the full width at half maximum (FWHM) $H_{\mathrm{pv}}$ of the peak profile to the FWHMs of the individual Lorentzian $\left(H_{\mathrm{L}}\right)$ and Gaussian $\left(H_{\mathrm{G}}\right)$ components. For the determination of instrumental broadening or in classical line profile analysis, the commonly used integral breadth $\beta_{\mathrm{pv}}$ is the ratio between integral intensity and amplitude. It can be easily derived by the mixing parameter $\eta$ and the FWHM of the pseudo-Voigt peak $H_{\mathrm{p} v}$ according to:

$$
\beta_{p V}=\frac{\pi H_{p V} / 2}{\eta+(1-\eta)[\pi(\ln 2)]^{1 / 2}}
$$

\subsubsection{Refinement and simulation methods}

The refinement of structural parameters conducted along this study was performed using Diffrac.Suite TOPAS by Bruker [29] applying the well known algorithm introduced by H.M. Rietveld [27] that fits the observed reflection intensities by least-square minimization. For cases, where the scattering intensities between the theoretical and observed pattern differ tremendously, the software offers the possibility to perform a so-called 'hkl'-fit by decoupling the structure factor coefficient from the linear addition. This method, the Pawley technique is a variation of the Le Bail-method [30], which uses the Rietveld-algorithm to estimate reflection intensities without a "structural" model, wherein the reflection positions are given by the generated unit cell of the phase. This is in particular useful, when a good fit is needed for irregular (asymmetric) profiles due to texture or dislocations or in the case of the presence of a phase with an unknown structure, the observed structure factor can be used for the structural model according to Rietveld-algorithm. The latter technique has been applied in this study. In the comparison with the convolutional fitting techniques (see below) all refinements done with the TOPAS software are referred to a Rietveld-type fitting in the discussion.

The simulation of the Cu-pattern with varying concentrations of coherent, intrinsic stacking faults was carried out with DIFFaX, a Fortran program, written by M. M. J. Treacy [31]. DIFFaX computes the average interference wavefunction scattered from each layer type occurring in a faulted crystal, by exploiting the recurring patterns found in randomized stacking sequences. In our simulations we used an infinite crystal size with a random sequence of intrinsic stacking faults in the [111] direction (ideal sequence in fcc: A-B-C-A) with a transition probability from 0.1 to $3.3 \%$ from $A$ to $B$ and vice versa. The reflection intensities in the calculated powder pattern where fitted with an analytical pseudo-Voigt function to determine their Fourier-coefficients as a function of the stacking fault probability $\alpha$.

Whole-powder-pattern-modelling (WPPM) was carried out with PM2K, a program, written and made freely available by $\mathrm{M}$. Leoni [32]. In contrast to the linear addition of coefficients, performed in the Rietveld-algorithm, PM2K fits the observed intensity of a powder pattern by an ab-inito Fourier-synthesis of a theoretical pattern with least-square minimization. The initial "structure model" is defined by the input lattice parameter of the phase and its indexed reflection peaks. As any (micro-)structural feature of the sample contributes an average interference wavefunction, the determination of their specific Fourier-coefficients can be used to convolve them in the Fourier-synthesis (for details see supporting information). In our fitting procedure, the neutron wavelength, sample displacement, initial lattice parameters and the Chebyshev background-polynomial parameters were adapted from the previous Rietveld-refinement and the instrumental broadening was determined as described above. The initial parameters for the 
defects analysis using PM2K were determined as described in the results and discussion section in detail.

\subsection{Transmission electron microscopy (TEM)}

A Philips CM200FEG microscope operated at $200 \mathrm{kV}$ and equipped with a field emission gun and the Gatan imaging filter was used for TEM. The coefficient of spherical aberration was $\mathrm{C}_{\mathrm{s}}=1.35 \mathrm{~mm}$. The information limit was better than $0.18 \mathrm{~nm}$. High-resolution TEM (HRTEM) images with a pixel size of $0.016 \mathrm{~nm}$ were taken at the magnification of $1083000 \times$ with a CCD camera.. Projected areas have been measured and equivalent diameters calculated for $1500-$ $4000 \mathrm{Cu}$ particles in each sample; in all cases the values of standard error of the mean diameter were $0.5 \mathrm{~nm}$ or less. Frequency distributions of $\mathrm{Cu}$ particle sizes fitted well to log-normal functions.

\section{Results and Discussion}

\subsection{Transmission electron microscopy}

All three catalysts have been subjected to a detailed TEM and HRTEM study. Images taken at moderate resolution (Fig. 1a-c) confirm the nanostructured nature of the composite catalysts. The $\mathrm{Cu}$ metal particles appear darker than the oxide phase and exhibit an approximately spherical shape. It is noted that the $\mathrm{Cu} / \mathrm{ZnO}$-catalysts are not classical supported systems with extended support particles that may have a distinct orientation with respect to the electron beam. $\mathrm{Cu} / \mathrm{ZnO}$ rather are bulk-catalysts with aggregates of $\mathrm{Cu}$ and oxide nanoparticles of comparable size. Thus, each image shows several dozens of $\mathrm{Cu}$ particles with a random orientation with respect to the electron beam. Because the TEM images do only show circular projections of the Cu particles, it can be safely concluded that the particle shape can indeed be approximated by a sphere and is not, for instance, a projection of aligned nano-rods. Thus, after reduction, the $\mathrm{Cu}$ particles in the $\mathrm{Cu} / \mathrm{ZnO}$-based catalysts exhibit an isotropic shape and a surface faceting that does not favor exposition of large terraces.

Evaluation of the $\mathrm{Cu}$ particle sizes based on a series of several images such as shown in Figure 1a-c yielded the particle size distribution (PSD) curves as shown in Figure 2. The PSD histogram was found to be relatively uniform (see supporting information) and can be fitted with a log-normal function, which is in agreement with earlier work $[22,16]$. From the PSD data, the volume weighted average $\mathrm{Cu}$ particle size has been calculated. It was $13.2 \pm 0.1 \mathrm{~nm}, 10.5 \pm 0.5$ $\mathrm{nm}$ and $11.0 \pm 0.2 \mathrm{~nm}$ for the catalysts CZ, CZA-1 and CZA-2, respectively (the \pm -value being the standard error, not the width of the PSD). This result is in agreement with the observation that the presence of the Al promoter leads to smaller $\mathrm{Cu}$ particles and a higher resistivity of the microstructure against sintering during reduction [33]. 

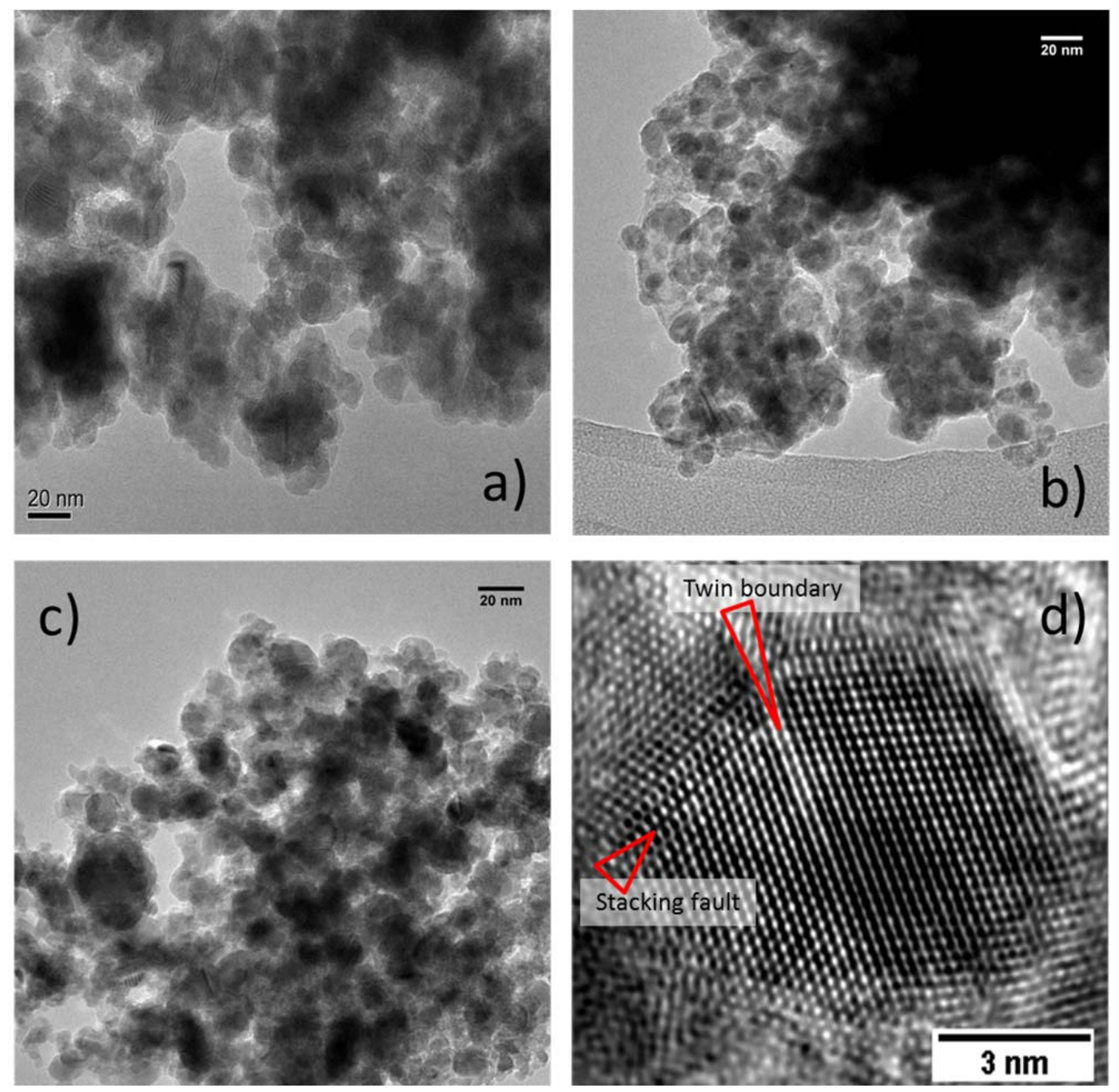

Figure 1: Representative TEM images of CZ (a), CZA-1 (b) and CZA-2 (c) taken at moderate magnification that were used for particle size evaluation. HRTEM image of a Cu nanoparticle in the sample CZA-1 that shows the presence of planar defects (stacking faults and twin boundaries, arrows).

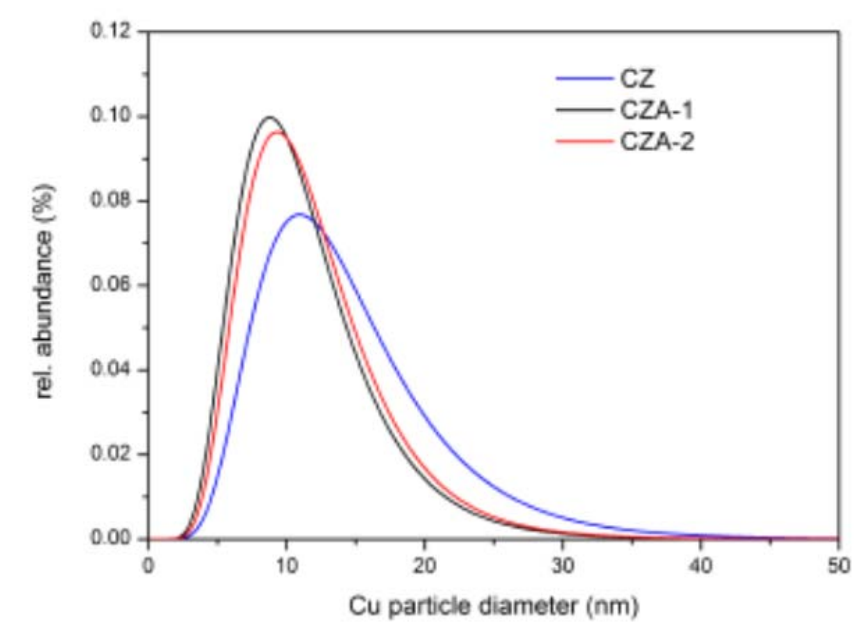

Figure 2: Log-normal fits of the particle size histograms obtained by evaluation of the TEM images of the three different catalysts (the histograms are presented as supporting information). 
The description of the catalyst's microstructure based on these direct observations obtained by TEM appears to be complete and unerring. However, several well-known pitfalls have to be taken into account and are briefly recalled in the following. First of all, it is important to consider that TEM is a local method. Care has to be taken to ensure that the aliquot of material transferred in the microscopy is representative of the whole batch of material under study. Also, the images taken need to be representative of the whole aliquot, e.g. by taking an adequate number of different TEM images at moderate resolution at different locations of the aliquot. $A$ selection of different TEM images used for the PSD evaluation of the sample CZA-1 showing different $\mathrm{Cu} / \mathrm{ZnO} / \mathrm{Al}_{2} \mathrm{O}_{3}$ aggregates of homogeneous microstructure is shown as an example in the supporting information. Concerning the former issue, it is important to assess the homogeneity of the sample by other complementary methods. This may include integral methods like diffraction to see if several phases are present (see below). But also other microscopy techniques of lower resolution like SEM can be useful to identify different types of material in a sample. EDX is especially important to detect local variations in the chemical composition that would indicate a heterogeneous sample. For the $\mathrm{Cu} / \mathrm{ZnO}$ catalysts studied here, a relatively homogeneous microstructure was confirmed by a TEM-EDX study. The results for sample CZA-1 are shown in the supporting information showing that the fluctuations of the average local composition of the different $\mathrm{Cu} / \mathrm{ZnO} / \mathrm{Al}_{2} \mathrm{O}_{3}$ aggregates are lower compared to comparable catalysts reported in literature [16].

Based on such validated TEM data, a high validity range of the log-normal distribution of the particle sizes can be achieved, which requires a reasonable maximum relative error. Romeo et al. stated, that for a number larger than 1000 arguments (particles) the relative error reaches its minimum [34]. To safely exclude improper statistics to affect the results, at least 1300 particles have been evaluated for each catalyst in this study. Another source of uncertainty is the presence of very small particles below $3 \mathrm{~nm}$. Their clear visibility in the TEM images is determined by contrast effects of the image generation and can be obscured leading to an underestimation of the lower-size tail of the PSD peak [35]. This effect is not only a function of the phase contrast between metal particle and support, but also depends on the quality of the used microscope and on the setting of the microscopy parameters for image taking [36]. Last, but not least the lower cut-off for the recognition of small particles critically depends on the eye of the operator. In particular in highly dispersed powder systems, the individual decision of the operator whether a small contrast variation is assigned to a particle or not will influence the average results. For example, in our group generally higher average volume-weighted particle sizes were obtained if the same TEM images were evaluated by a less experienced co-worker in all conscience. It is noted that the relative difference observed between different samples were usually confirmed by all operators. Thus, care has to be taken when comparing TEM-derived data reported in different literature studies, in particular if absolute values are concerned. The relative trend of a sample series determined under identical conditions by the same operator, however, can be regarded as reliable provided the material is homogeneous and the relative contrast of particles and support is comparable. However, it is certainly advisable in all cases to complement the size information determined by TEM by integral methods. Concerning our three $\mathrm{Cu} / \mathrm{ZnO}$-based catalysts, we conclude that both alumina-promoted CZA catalysts show relatively uniform PSDs with similar narrow widths, while the unpromoted $C Z$ catalyst exhibits significantly larger Cu particles and a slightly wider PSD.

Additional information on the microstructure of the catalysts can be obtained from high resolution images. Due to the intrinsically bad statistics of HRTEM data, this information should not be used for quantification or extrapolation. However, atomic resolution images such as shown in Figure 1d enable inspection of the $\mathrm{Cu}$ lattice in individual particles concerning the presence of extended defects. Planar defects, stacking faults and twin boundaries, are found quite frequently in most investigated Cu particle of all catalyst samples. An example is shown in Figure 1d. These defects originate from imperfections in the stacking sequence of the close packed (111) layers and their influence on the diffraction peak profiles will be discussed below in 
more detail. Other HRTEM images of these and related catalysts can be found in [16, 19, 22].

\subsection{Phase identification and Rietveld refinement of the neutron diffraction data}

The phase analysis and crystal structure refinement using the Rietveld method will be treated here on basis of the neutron diffraction patterns. The evaluation of the XRD results will be discussed later in section 3.5 that reverts on the results presented in this section. The advantage of neutron vs. X-ray diffraction is that the intensity scattered at the nuclei does not decay with $2 \theta$, while X-rays interact with the spatially extended electron clouds of the atoms. Thus, higher intensity and a larger number of higher order peaks are usually detected at large $2 \theta$ using neutrons. This is in particular important for a more reliable refinement of highly symmetric structures with only few reflections such as the fcc-Cu structure in our catalysts.

The neutron diffraction patterns of the four samples are shown in Figure 3. All observed peaks do either belong to fcc-Cu or to Wurzite-type $\mathrm{ZnO}$ indicating the absence of other crystalline phases. It can be seen at first sight, that the Cu peaks of the catalysts are significantly broadened compared to those of C-ref. Furthermore, clear differences exist in the crystallinity of the $\mathrm{ZnO}$ component. As a first step of (micro-)structural analysis, the Rietveld-refinement procedure [27] was applied to the raw patterns. This widely-applied method [12] is used to refine crystal structure parameters and to determine the phase fractions in multi-phase samples. The Rietveld method fits the whole pattern simultaneously by calculation of the expected intensity at a given $2 \theta$ and least-squares minimization of the difference between calculated and observed intensities through variation of the atomic structure parameters including atomic coordinates, Debye-Waller-factors and site occupancy. This method is very powerful and preferred to single peak and pattern decomposition methods in particular if the pattern is affected by peak overlapping. The best fits of the four samples are graphically represented in Figure 3 and the refined structural details are shown in the supporting information. The lattice parameters of the Cu phases in the catalysts were $361.32 \pm 0.06 \mathrm{pm}, 361.26 \pm 0.12 \mathrm{pm}$ and $361.19 \pm 0.15 \mathrm{pm}$ for CZ, CZA-1 and CZA-2, respectively (the \pm -values being $3 \times$ the estimated standard deviation of the Rietveld fit). These values are slightly lower than the lattice parameter refined for C-ref, which was $361.413 \pm 0.012 \mathrm{pm}$, and close to the literature value. The Cu lattice compression in the catalysts samples is small but significant and consistently observed in all three catalyst samples. It is likely an effect of the defective nature of the nanoparticles and/or the intimate interfacial contact of $\mathrm{Cu}$ and the $\mathrm{ZnO}$-based oxide phases.

An important difference of the three catalysts is the crystallinity of the oxide phase. While in $\mathrm{CZ}$ the $\mathrm{ZnO}$ peaks are clearly present, they are much weaker in CZA-2, finally the pattern of CZA-1 can be satisfactorily fitted without any contribution of an oxide phase despite the similar $\mathrm{ZnO}$ content of all three samples. This is a results of the different preparation method as the suppression of precursor ageing as well as the presence of the $\mathrm{Al}_{2} \mathrm{O}_{3}$ promoter lead to a more amorphous $\mathrm{ZnO}$ component [22]. No crystalline Al-containing phase can be detected in the patterns of the two CZA samples. 


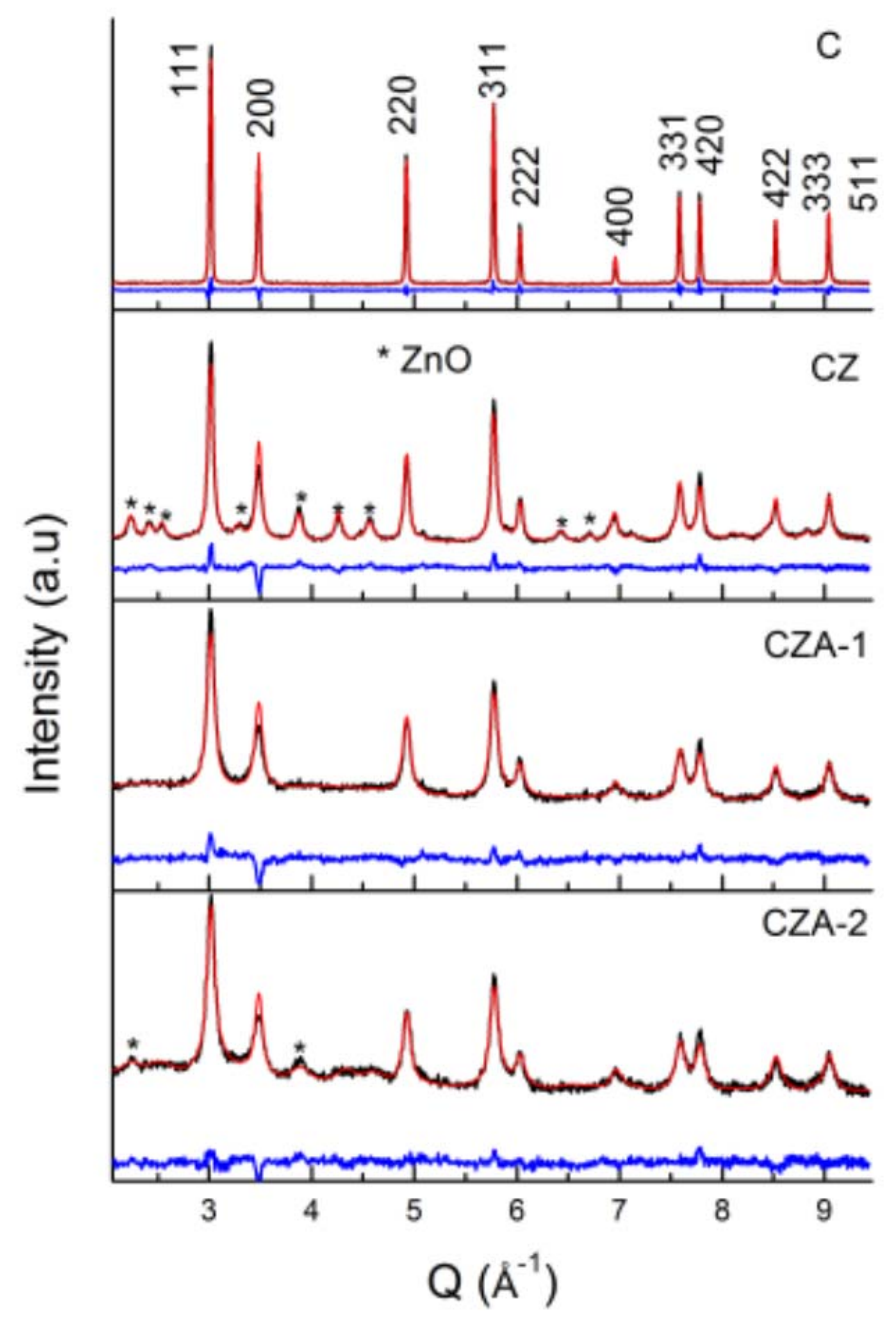

Figure 3: Rietveld plots of the C-ref material and the samples CZ, CZA-1 and CZA-2 and (black - experimental data; red - calculated pattern; blue - difference curve). The peaks due to fcc-Cu are indexed in the uppermost panel, those due to Wurzite-type $\mathrm{ZnO}$ are marked "*”. Note the systematic deviations in the difference curves of the three catalysts.

The observed differences in peak widths are typically attributed to small domain size and/or lattice strain effects. Both effects can be discriminated by the $2 \theta$-dependence of the peak widths, which is stronger for strain broadening. The peak widths and profiles are determined during the Rietveld refinement foremost with the goal to model the experimental pattern as good as possible with the lowest possible number of refined parameters. Thus, the $2 \theta$-dependency of the peak profile parameters is usually described using simple and steady functions with only a few parameters. Although these restrictions are certainly not optimal for a microstructural analysis of the peak profiles, most standard-Rietveld refinement programs have modules included that compare these few parameters with the instrumental resolution determined on a strain-free and macrocrystalline standard pattern and deliver a physical interpretation of this data in addition to the average crystal parameters. This procedure allows for estimation of the domain size as well as of the lattice strain, but it has to be taken into consideration that the Rietveld routine forces all different forms of peak broadening into this simplified scheme, regardless of its real physical origin. However, if only isotropic strain and isotropic size effects are present, i.e. the peak profiles can indeed be described reasonable well by a steady function over the whole 20-range, this method can deliver accurate results. It is noted that some Rietveld programs additionally 
allow the use of different $2 \theta$-dependencies for different families of hkl, i.e. they take anisotropy of the size (shape) of the domain and of the lattice strain into account.

The Rietveld software package used for this study makes use of the so-called double-Voigt approach to differentiate between size an strain effects [37]. The crystallite domain size is given as an average volume-weighted column length based on the integral breadths of all reflections and amounts to $9.9 \pm 1.2 \mathrm{~nm}, 5.8 \pm 0.6 \mathrm{~nm}$ and $7.1 \pm 1.5 \mathrm{~nm}$ for CZ, CZA-1 and CZA-2, respectively. These values are smaller than the particle sizes determined by TEM, but the relative trend of the three samples is in good agreement between both methods. Generally, a smaller size is expected for the coherently scattering domains determined by diffraction compared to the particle size determined by TEM as one particle might consist of several domains. This size discrepancy between TEM-size and diffraction-size can be related to the observed presence of planar defects in the $\mathrm{Cu}$ particles, because individual particles can be divided into domains by stacking faults and twin boundaries (see below). The lattice strain $\Delta \mathrm{d} / \mathrm{d}_{0}$ is extracted from the Rietveld fits as a Gaussian distribution of the lattice spacings around an average value $d_{0}$. Generally low values are observed for the catalysts and $0.05 \pm 0.02 \%, 0.16 \pm$ $0.06 \%$ and $0.07 \pm 0.05 \%$ was found for CZ, CZA-1 and CZA-2, while an even lower value of $0.018 \pm 0.004 \%$ was detected for C-ref.

While Rietveld refinement is the preferred method to determine the (crystalline) phase composition and any details of the average ideal structure (like the Cu lattice parameter), the quantification of deviations from the ideal structural model that was used to calculate the expected intensities has to be treated with care. This is because the strain and size values given above do usually not consider effects of anisotropy. Anisotropic peak broadening can be induced by different sources such as anisotropic particle shape, anisotropic strain and defects. Careful inspection of the difference curves of the best Rietveld fits shown in Figure 3 reveals clear indications for anisotropic effects in the three catalyst samples. It can be seen that the Rietveld routine does not succeed in accurately fitting all peak profiles of the Cu phase in the catalysts. Systematic deviations exist between experiment and calculated pattern as a result of the refinement yielding the best compromise for the peak profiles using the "isotropic" functions available. These deviations include an underestimation of the intensities of the 111, 311 and 420 peaks, while the 200 is overestimated and shows a negative residual in the difference curve. A similar but less clear effect is also observed for the higher order 400 peak, best seen in the pattern of $C Z$. Such systematic deviations are not observed for the macrocrystalline C-ref material. These deviations contain important information on the $\mathrm{Cu}$ micro- and defect structure that is not reflected in the Rietveld refinement results and will be evaluated in more detail in the following sections. Due to the isotropic cubic crystal structure of $\mathrm{Cu}$ and the approximately spherical shape of the particles seen in TEM, anisotropic size or strain effects can be excluded as the source of these deviations. As discussed by Warren [38] and later demonstrated by Balogh et al. [39] significant amounts of intrinsic stacking faults as well as twin boundaries can cause extensive anisotropic and nonuniform peak profile changes, which cannot be attributed neither to size nor to strain broadening. As the defective nature of our investigated material is known from earlier work $[16,18]$ and from the HRTEM results presented in Figure 1d, planar defects are the likely origin of these effects in the methanol synthesis catalyst. Tsybula et al. point out, that in case of nanocrystalline materials, local imperfections in the regular structure can be considered as elements of the nanostructure, being integral or specially imposed parts of the latter [12].

\subsection{Pattern decomposition methods}

Evaluation of a diffraction pattern using the pattern decomposition method is based on the description of the experimental pattern by the sum of several analytical functions for the peaks (here: pseudo-Voigt functions) and the background (e.g. a polynomial). Contrary to the Rietveld method, no structural model restricting the relative peak positions and intensities and no pre- 
defined form of the 20-dependency of the peak profiles is used. Thus, the primary result of pattern decomposition is a number of independent peak profiles containing 20-position, width and shape parameter. This method can be applied to single peaks, sections of the pattern or the whole data range. While extraction of structural information (lattice parameter) from this data is much less accurate compared to the Rietveld method, the peak profile parameters contain all information due to anisotropic effects needed for a full microstructural analysis. A comparison of Rietveld fit and pattern decomposition is shown in Figure 4 for the 331 and 420 peaks of the sampe CZA-1.

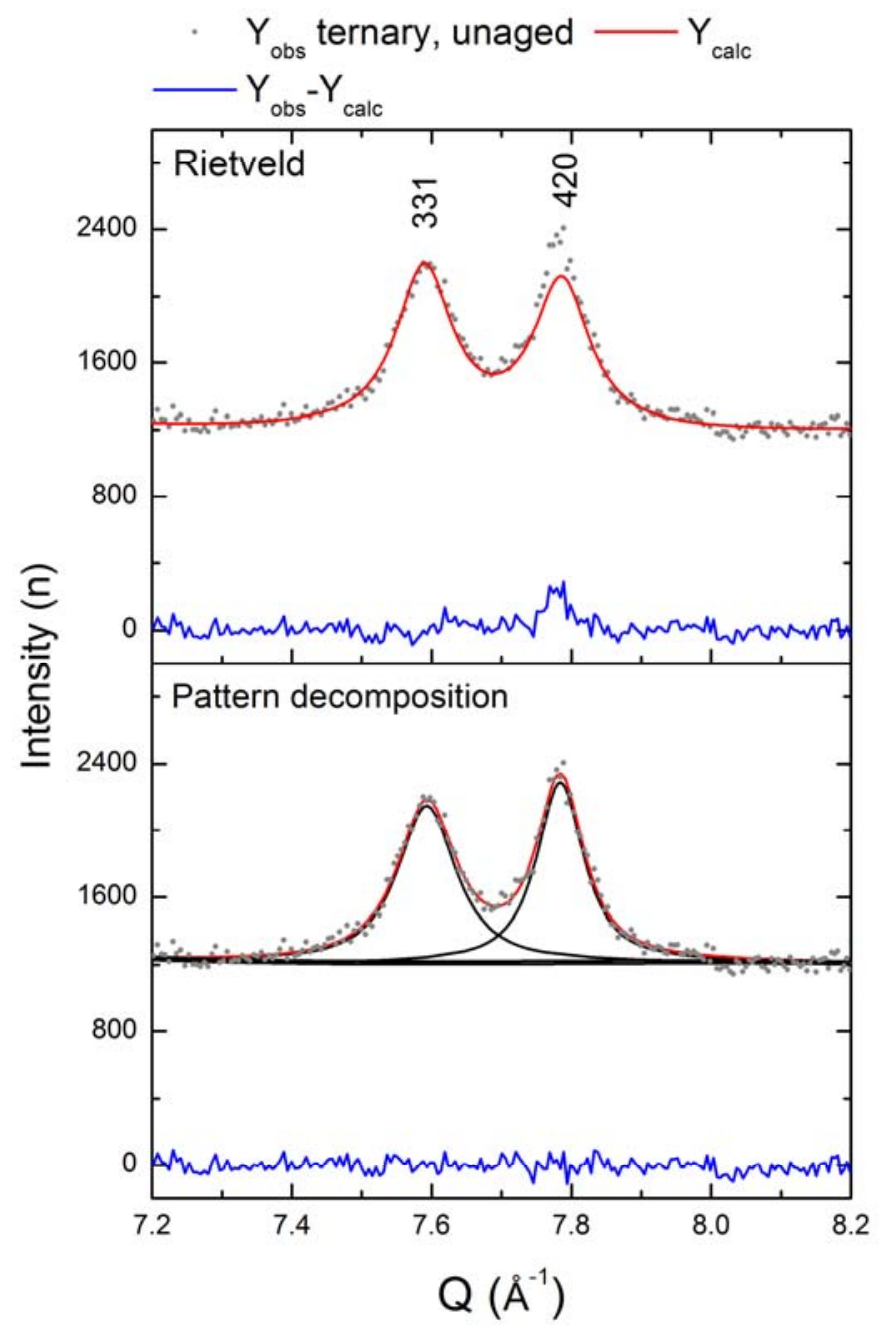

Figure 4: Comparison of the fitting results of the 331-420 peak doublet of CZA-1 using the Rietveld method and the model-free pattern decomposition method. The larger deviations between calculated and experimental data in the upper panel are due to the restrictions implied by the ideal structural model used for the refinement.

These two peaks, which due to their high $Q$ and often low intensity are only rarely recorded in XRD patterns of fcc nanomaterials, show an anisotropic broadening. The 331 at lower scattering angle is significantly broader compared to the neighboring 420 peak. The Rietveld routine applied in this study tries to fit these peaks with similar widths and with interdependent positions given by the $\mathrm{Cu}$ lattice parameter. As a result, the intensity of the sharper 420 peak is not properly described in the calculated pattern. Additionally, only the outer tails of the doublet are adequately fitted while the inner tails are underestimated. As a consequence, the calculated spacing of the peaks is larger than the experimentally observed one. Naturally, the model-free pattern decomposition approach with its higher numbers of parameters and less restrictions allows fitting this feature more satisfactorily. It is noted, however, that this method generally 
deals much worse with peak overlapping and some instrumental artifacts like the zero-shift of the Q-scale that can be treated much better with the Rietveld method. For the analysis of the catalysts in this study, a combination of Rietveld and pattern decomposition was used to mitigate these problems. The zero-shift and the peak profiles of the $\mathrm{ZnO}$ phase (if present in crystalline

form) were determined by Rietveld refinement and used for correction of the raw peak profiles of the $\mathrm{Cu}$ phase extracted from pattern decomposition. Furthermore, the instrumental contribution to the peak profiles was deconvoluted from the experimental data as described in the supporting information.

\subsubsection{Application of the Scherrer equation}

As mentioned in the introduction, the application of the Scherrer equation is probably the most popular method of extracting size information from diffraction data, but it is subjected to several limitations that need to be taken into account for a physical interpretation of the results. $P$. Scherrer related the integral breadth $\beta$ of a diffraction line to the finite size of the diffracting crystals normal to the diffracting planes, also named column length $L$, or apparent crystal size, by the expression [40-41]:

$$
L=\frac{\lambda}{\beta \cos \theta}
$$

The "apparent" crystal size $D_{\text {eff }}$ can be obtained from the column length $\mathrm{L}$

$$
D_{\text {eff }}=K \cdot L
$$

wherein $\mathrm{K}$ is a form-factor ('Scherrer-constant'), which takes the size distribution of different reflecting planes (e.g. for spheres) into account. It has to be considered that a distribution of crystallite sizes will affect the breadth of a reflection in different ways [23,38,42-43]. In case of a certain size-distribution (e.g. Log-Normal) a modified Scherrer-Constant must be used. Langford et al. described, that the breadth of diffraction maxima for crystallites having the same shape vary systematically with the indices of reflection. If a certain crystallite shape is known or assumed from additional methods, adequate values might be assigned to $K$ [43]. For this study, a value of $K=0.89$ for spherical shape was used based on the TEM results (while $K=0.9$ is recommended for cubic crystals and also in case of unknown crystal shape [23]). Applicability of Scherrer-Formula is restricted to pure physical broadening solely due to crystallite sizes below approximately $100 \mathrm{~nm}$. Accordingly, the results obtained for the macrocrystalline C-ref sample should not be physically interpreted.

The crystallite domain sizes calculated by application of the Scherrer equation on the individual peak profiles obtained by pattern decomposition are presented in Table 2. It can be seen that the anisotropy of the peak profiles is reflected in variations of the calculated domain sizes. For example, within the sample CZA-1 the resulting sizes vary between 1.7 and $4.8 \mathrm{~nm}$ obtained for the 400 and 111 peak, respectively. This is a result of the anisotropic peak profiles already observed in the difference plots of the Rietveld refinements. Accordingly, the Scherrer approach predicts for all samples a larger domain size in the [420] direction compared to [331] as a result of the sharper peak profile of the former reflection, as seen in Figure 4 . These variations shall not be interpreted as anisotropy of the particle shape, which as seen by TEM is rather isotropic. The average values are smaller than the ones obtained by the Rietveld analysis, probably because the strain contribution to the peak width is neglected in the Scherrer analysis. Even the size trend CZ > CZA-2 > CZA-1 that has been consistently found by Rietveld analysis and TEM is now changed to CZ > CZA-1 > CZA-2, which might be an artifact due to the slightly differences in strain.

The values in Table 2 are an example how important microstructural information can be easily overlooked if the analysis is solely based on Scherrer evaluation of a single arbitrarily 
chosen peak. It must be kept in mind that such one-peak evaluation gives only a crude estimation of the domain sizes, but does not reflect the whole microstructural information from the peak profiles and cannot account for anisotropic effects. The example of the $\mathrm{Cu} / \mathrm{ZnO}$-based catalysts furthermore shows that the resulting size domain information should not be prematurely interpreted as "particle size".

Table 2: Results of the "apparent crystal size" determined by application of the Scherrer formula on the integral breadth of each individual peak resulting from pattern decomposition $(\mathrm{K}=$ 0.89). The averaged "apparent crystal size" is given in the last column. All values in nm.

\begin{tabular}{|l|l|l|l|l|l|l|l|l|l|l|l|}
\hline $\mathrm{D}_{\text {hkl }}$ & 111 & 200 & 220 & 311 & 222 & 400 & 331 & 420 & 422 & $333 / 511$ & $\mathrm{D}_{\text {ave }}$ \\
\hline C-ref & 16.62 & 15.67 & 18.12 & 18.61 & 19.01 & 17.68 & 18.07 & 18.92 & 18.15 & 17.42 & 17.83 \\
\hline CZ & 6.72 & 4.89 & 6.38 & 6.13 & 6.93 & 3.85 & 4.91 & 6.16 & 4.61 & 5.48 & 5.61 \\
\hline $\begin{array}{l}\text { CZA- } \\
1\end{array}$ & 4.79 & 3.99 & 4.52 & 4.67 & 4.81 & 1.65 & 3.46 & 4.29 & 3.80 & 4.06 & 4.00 \\
\hline $\begin{array}{l}\text { CZA- } \\
2\end{array}$ & 4.08 & 3.30 & 4.25 & 3.96 & 4.07 & 1.54 & 3.21 & 3.89 & 3.85 & 3.47 & 3.56 \\
\hline
\end{tabular}

\subsubsection{Williamson-Hall Methods}

In contrast to the Scherrer analysis, the evaluation of peak profiles obtained by pattern decomposition using the method of Williamson and Hall is able to discriminate between strain and size effects and yields average values of microstructural parameters [42]. In the case of negligible instrumental broadening (e.g. for certain synchrotron data or for a semi-quantitative evaluation of a series of samples measured on the same machine) the application can be straightforward, but otherwise great care must be taken to model or measure instrumental contribution very accurately.

The method assumes that, if the reflection profiles can be satisfactorily fitted by a pure Lorentzian function, the total physical broadening of a reflection profile is a linear addition of 'size' $\left(\beta_{\mathrm{S}}\right)$ and 'strain' $\left(\beta_{\mathrm{D}}\right)$ effects. The so-called 'Williamson-Hall plot' is a good starting point for a quantitative description of the microstructure. For isotropic effects, the plot of $\beta_{\mathrm{S}+\mathrm{D}}(\cos \theta) / \lambda$ (in $\mathrm{nm}^{-1}$ ) vs. $2 \sin (\theta) \lambda$ (in $\mathrm{nm}^{-1}$ ) should results in a straight line. The crystallite domain size $D_{\mathrm{hkl}}$ can be derived from the intercept and the mean residual strain $\varepsilon_{\mathrm{hkl}}$ from the slope [44].

However, such a plot does hardly give straight lines for the three catalyst samples as shown in Figure 5. Only the macrocrystalline C-ref sample shows an almost linear distribution of the data points indicating that Cu-ref is the "most isotropic" material in the study as expected and in agreement with the other methods applied. Due to the large scattering, the Williamson-Hall plots of the other samples should not be evaluated in detail. The large deviation from linear regression can be interpreted as anisotropic behavior due to non monotonous 20-dependence of the diffraction profile widths [45]. Such anisotropy in Williamson-Hall plots was attributed to lattice dislocations/imperfections [45-46] and/or twin faulting $[38,47]$. 


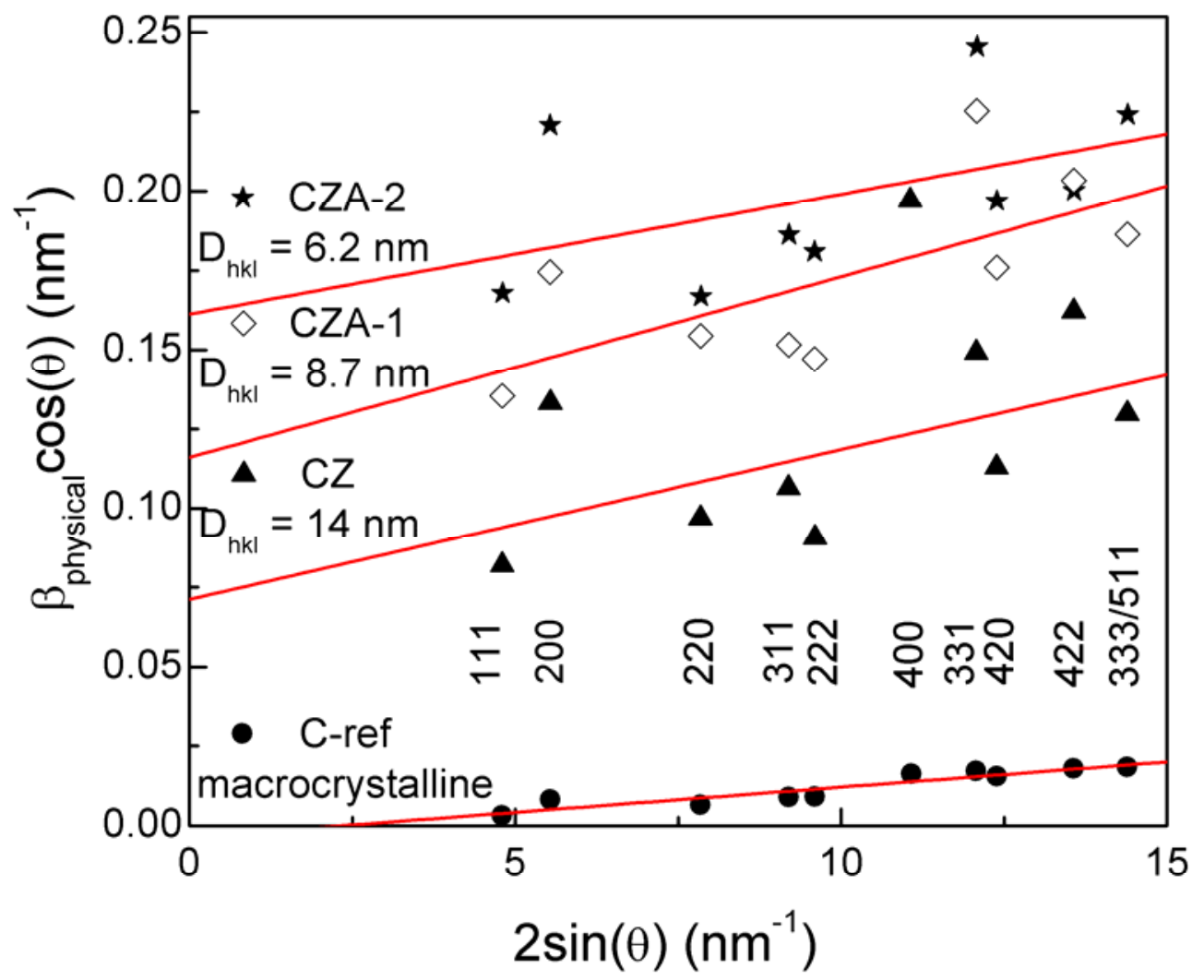

Figure 5: Williamson-Hall plots of C-ref and the three catalyst sample CZ, CZA-1 and CZA-2. The deviations of the three catalysts from linearity are discussed in detail in the text.

Thus, the Williamson-Hall plots in Figure 5 are a graphical representation of the anisotropy that has been observed already in the systematic deviations of the difference curve of the Rietveld refinement and in the scattering of the domain size values obtained by the Scherrer analysis. Close inspection of Figure 5 reveals that the deviation from linear behavior is in particular caused by an extraordinary broadening (a too high value of $\beta(\cos \theta)$ ) of the 200,400 and 331 peaks - those peaks that tend to be overestimated by the Rietveld algorithm and that deliver the lowest size values upon application of the Scherrer equation. Note that values for the 400 of the catalysts CZA-1 and CZA-2 are larger than 0.5 and not shown in Figure 5 . If an attempt is made to quantify size and strain despite the non-linearity of the plots (red lines in Fig. 5 , excluding the 400 peaks), crudely estimated size values are obtained that are larger than the Scherrer results and now fall closer to the results obtained by Rietveld refinement or TEM analysis (size data given in Figure 5, strain in supporting information). However, the results still suggest that the crystallites in CZA-2 are smaller than in CZA-1.

Ungar and Borbely introduced a modified Williamson-Hall plot, which they applied successfully on the line profile analysis of an ultrafine grain $\mathrm{Cu}$ specimen [48] that can account for the anisotropic behavior using an additional fundamental parameter, the contrast factor $C$. The average contrast factor $\bar{C}$ is a weighted average of individual contrast factors, which is obtained by averaging over all permutations of the Miller indices of a particular reflection. In the modified Williamson-Hall plot the FWHM $\Delta \mathrm{K}$ is plotted versus a term $K \sqrt{\bar{C}} . \Delta K^{\mathrm{D}}$ is a strain term contributing to line broadening and $D$ is the average grain or particle size $(K=2 \sin \theta / \lambda$, and $\Delta K=$ $2 \cos \theta(\Delta \theta) / \lambda$ ) [48]. Ungar and Tichy point out, that the numerical calculated $C$ factors of copper 
were averaged assuming equal dislocation population in each possible slip system and equal probability of edge and screw dislocations [46]. Numerical values for different slip systems as well as average $\bar{C}$ factors are given in the literature $[48,46]$. The values used for $C$ in this study were calculated according to ref. [46]. For sake of conciseness the reader is referred to the literature $[46,48-50]$ for mathematical derivation and theoretical background. As the correlation is for spherical particles and the slope is fitted over several orders of reflections, the averaging Scherrer-factor of 0.9 (log-normal distributed spheres) was used in Eq. (6). The modified Williamson-Hall plots of the three catalyst sample are shown in Figure 6.

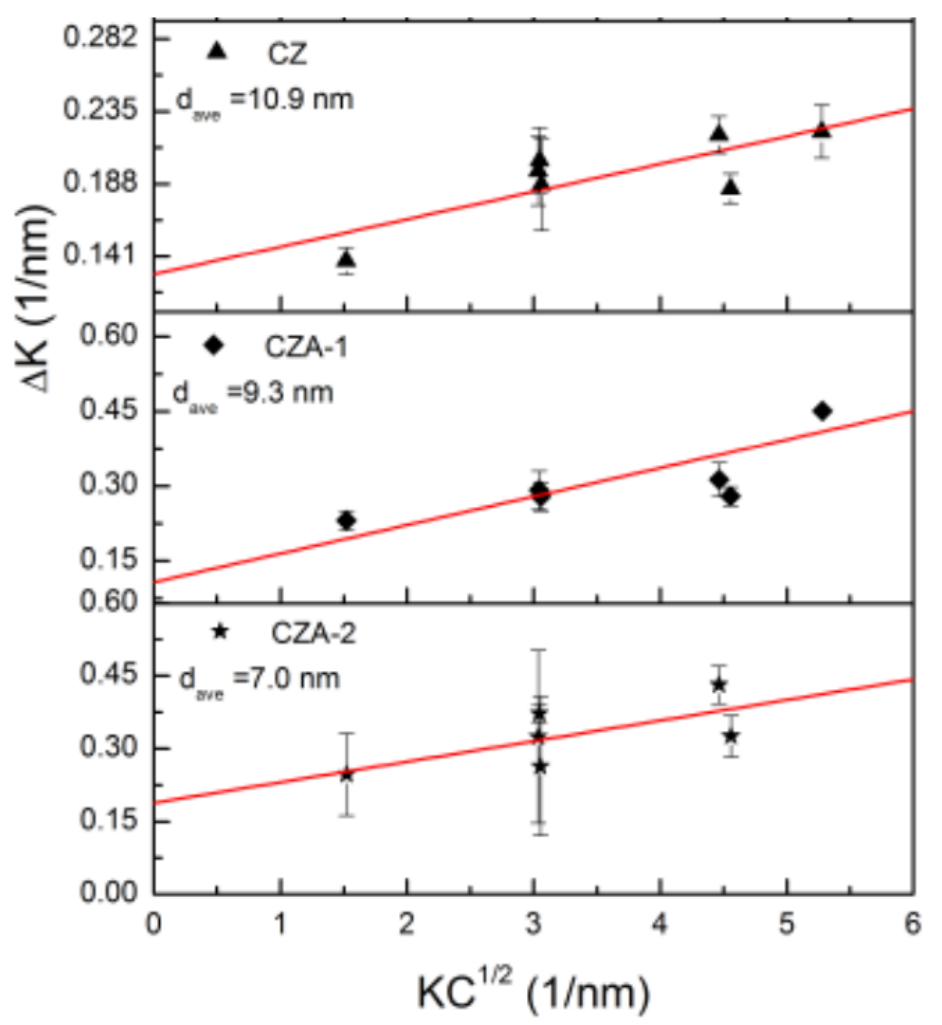

Figure 6: Modified Williamson-Hall plots of the three catalysts samples CZ, CZA-1 and CZA2.

It can be seen that the modified Williamson-Hall method deals much better with the anisotropy of the experimental peak profiles and that the deviation from linearity is less compared to the classical Williamson-Hall plots. Concerning the size data, the numerical results obtained from this method are slightly larger and can be considered as more reliable, but have no influence on the relative order of the catalysts.

The systematic deviations in the classical Williamson-Hall analysis and the fact, that the modification to take the effect of defects into account, delivers less deviation are additional indications that the microstructures of the methanol synthesis catalysts are affected by structural defects. The anisotropy induced by stacking faults in an fcc lattice is discussed in the following section in detail, concerning the effect of and twin boundaries and line defects like dislocations, the reader is referred to the supporting information for a brief treatment or to the literature cited there.

\subsection{Anisotropy due to stacking faults}

$\mathrm{Cu}$ is known to form stacking faults in direction of the crystallographic [111] direction [38,51-52], the ideal stacking fcc sequence being A-B-C-A-B-C. By addition of an additional $A$ layer one achieves a sequence of A-B-C-A-B-A-C-A-B - an extrinsic stacking fault. According to Warren 
[38], extrinsic stacking faults do not contribute significantly to peak broadening; therefore only intrinsic stacking faults (missing $C$ layer, A-B-C-A-B-A-B-C-A) are considered here. If an intrinsic stacking fault occurs, the fcc structure becomes locally isostructural to the hcp structure with a section of an $A-B-A-B$ stacking sequence. The $A$ layer is displaced to $B$ by $x=2 / 3, y=1 / 3$ and $z=$ 1 and $B$ to $A$ by $x=1 / 3, y=2 / 3$ and $z=1$. This is a known source of anisotropic peak broadening as observed in the neutron diffraction data of the three catalyst samples. It is described in the literature and in many crystallography textbooks [38,53] that stacking faults affect those components of the peak, for which $h+k+I=3 N \pm 1$, while components with $h+k+I=3 N$ are not affected. As an example, of the eight components of the highest 111 peak, six will be affected, while two remain unaffected. (The multiplicity of $\{111\}$ is eight and the peak contains intensities diffracted at (111) and (-1-1-1), which remains unaffected, and at (-111), (1-11), (111), (-1-11), (-11-1), (1-1-1), which will be affected.) To illustrate these two groups, the (111) and (-111) planes are drawn into a HRTEM image of a typical faulted Cu particle [54] in Figure 7 representing peaks that are unaffected and affected by stacking faults.
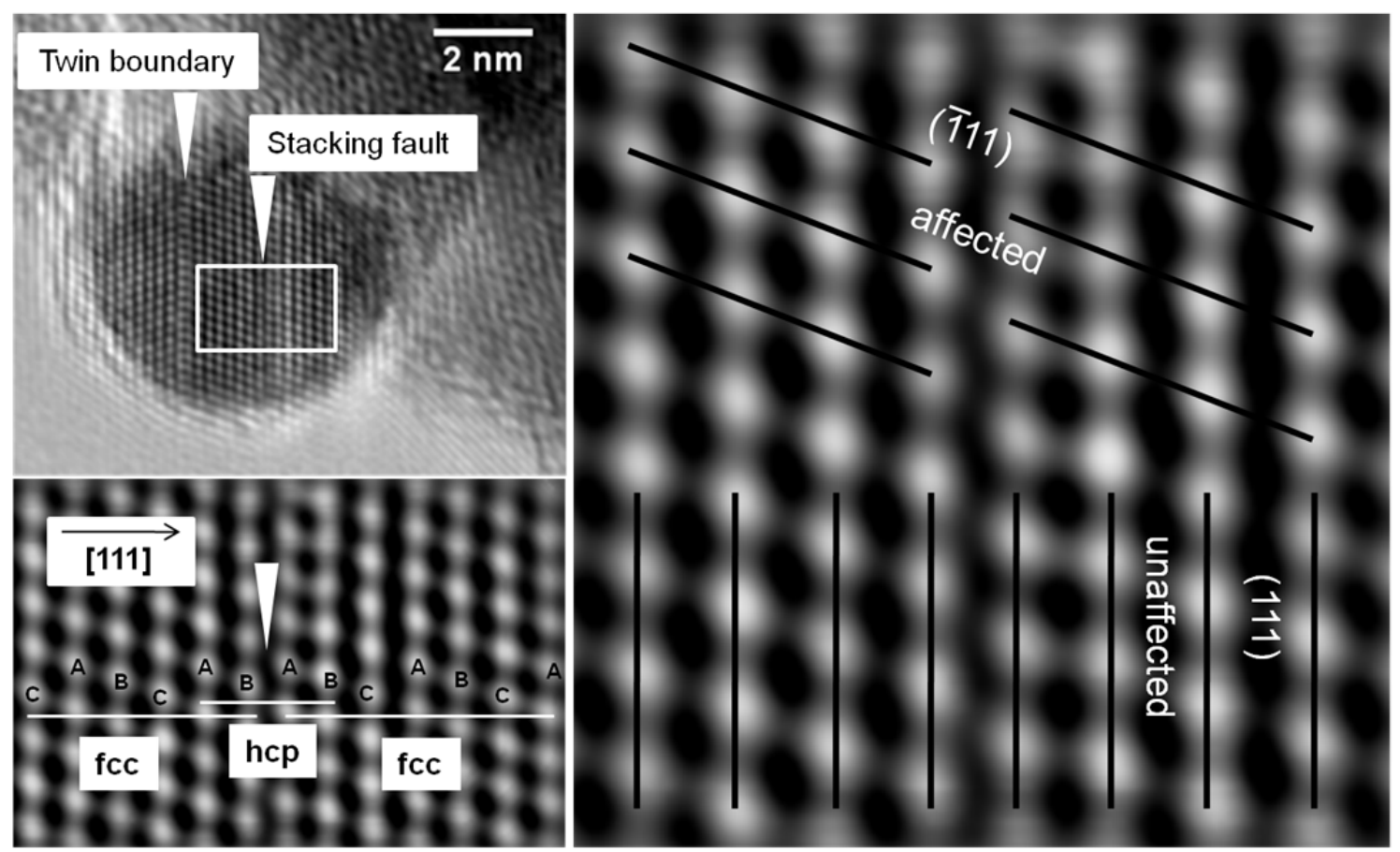

Figure 7: HRTEM image of a typical faulted $\mathrm{Cu}$ nanoparticle in a Cu/ZnO-based methanol synthesis catalyst. The close-up of the $\mathrm{Cu}$ lattice shows the stacking sequence of the (111) layers around the intrinsic stacking fault (left hand side) and exemplifies the different effects on the (111) and (111) components of the 111 diffraction peak.

In practice, this means that six components of the 111 peak will be shifted and broadened and form a shoulder at the higher angle tail of the 111 peak. This type of peak broadening will be most pronounced for those peaks that do not contain any contribution that remains unaffected, which are the 200 (6 components broadened and shifted to lower angles), the 400 (6 components broadened and shifted to higher angles) and the 331 (6 components broadened and shifted to lower angles and 18 components broadened and shifted to higher angles). Indeed, these three peaks that show the largest deviation from the linear behavior in the Williamson-Hall plots and deliver the lowest domain sizes in the Scherrer-analysis. Based on these qualitative considerations, also the systematic deviations in the Rietveld residual can be understood. For example, Figure 4 shows the inability of the Rietveld algorithm to adequately describe the peak profiles of the 331 and 420 peaks based on the ideal fcc model and an isotropic evolution of the peak width with $2 \theta$. As a result of stacking faults, a net shift of the peaks towards each other (12 components of the 420 will be shifted to lower angle while 12 
components will remain unaffected) and a larger width of the 331, which does not contain any unaffected components, can be expected. Indeed the experimental data clearly shows a narrower peak of the 420 resulting in a higher peak maximum compared to the 331 . Furthermore, the extra intensity at the inner tails of the peak doublet indicates that the true peak positions are closer together on the $2 \theta$-scale than calculated by the Rietveld software.

As shown above, the model-free pattern decomposition method can be used to analytically describe these small but systematic differences in the peak profiles. In a recent paper, we have applied such pattern decomposition to determine the peak positions and relative d-spacings of the 111 and 200 in comparison to the 222 and 400 for a series of Cu/ZnO-based methanol synthesis catalysts [18]. It was found that both pairs are shifted into opposite directions (111 and 200 towards and 222 and 400 away from each other) as is expected as a result of the presence of stacking faults. The deviation from the ideal value was used to quantify the stacking fault probability in the catalysts according to simple equations introduced by Warren [38]:

$$
\begin{aligned}
& \Delta\left(2 \theta_{200}-2 \theta_{111}\right)^{\circ}=\frac{-90 \sqrt{3} \alpha}{\pi^{2}}\left(\frac{\tan \theta_{200}}{2}+\frac{\tan \theta_{111}}{4}\right) \\
& \Delta\left(2 \theta_{400}-2 \theta_{222}\right)^{\circ}=\frac{-90 \sqrt{3} \alpha}{\pi^{2}}\left(\frac{\tan \theta_{400}}{4}+\frac{\tan \theta_{222}}{8}\right)
\end{aligned}
$$

where $\alpha$ is the stacking fault probability. As a result of stacking fault determination according to Eq.(8), a linear relation between stacking fault probability and the specific activity of the exposed Cu surface area was found. The stacking fault probabilities $\alpha$ of C-ref, CZ, CZA-1 and CZA-2 were $0.0018,0.0061,0.0050$ and 0.0155 , respectively [18]. This quantification is capable of finding reliable trends within a series of samples, but the absolute values suffer from the fact that it is solely based on the peak positions and neglects additional information from the peak widths and shapes. Velterop et al. [55] pointed out that the relation between peak maximum shift and stacking fault probability deviates from linear behavior above $\alpha=0.025$, because the peak positions in the convoluted raw data are dominated by the unaffected components. Herein, we report an attempt to additionally use the full peak profile information for such quantification. The profiles of deconvoluted $\mathrm{Cu}$ peaks that are affected by stacking fault broadening can be simulated using the DIFFaX software [31]. The profiles expected for three different stacking fault concentrations are shown in the first row in Figure 8 for selected reflections. These profiles are generally much narrower than the experimental peaks, because in the simulation no additional sample or instrumental broadening was considered. The different evolution of the peak profiles with increasing stacking fault concentration can be seen. For instance, the 111 peak (222 peak) develops a shoulder at the higher (lower) angle side, while the 200 peak (400 peak) is symmetrically broadened and shifted to lower (higher) angles. However, for the 220 for instance no such clear trend is observed (Fig. 8). 


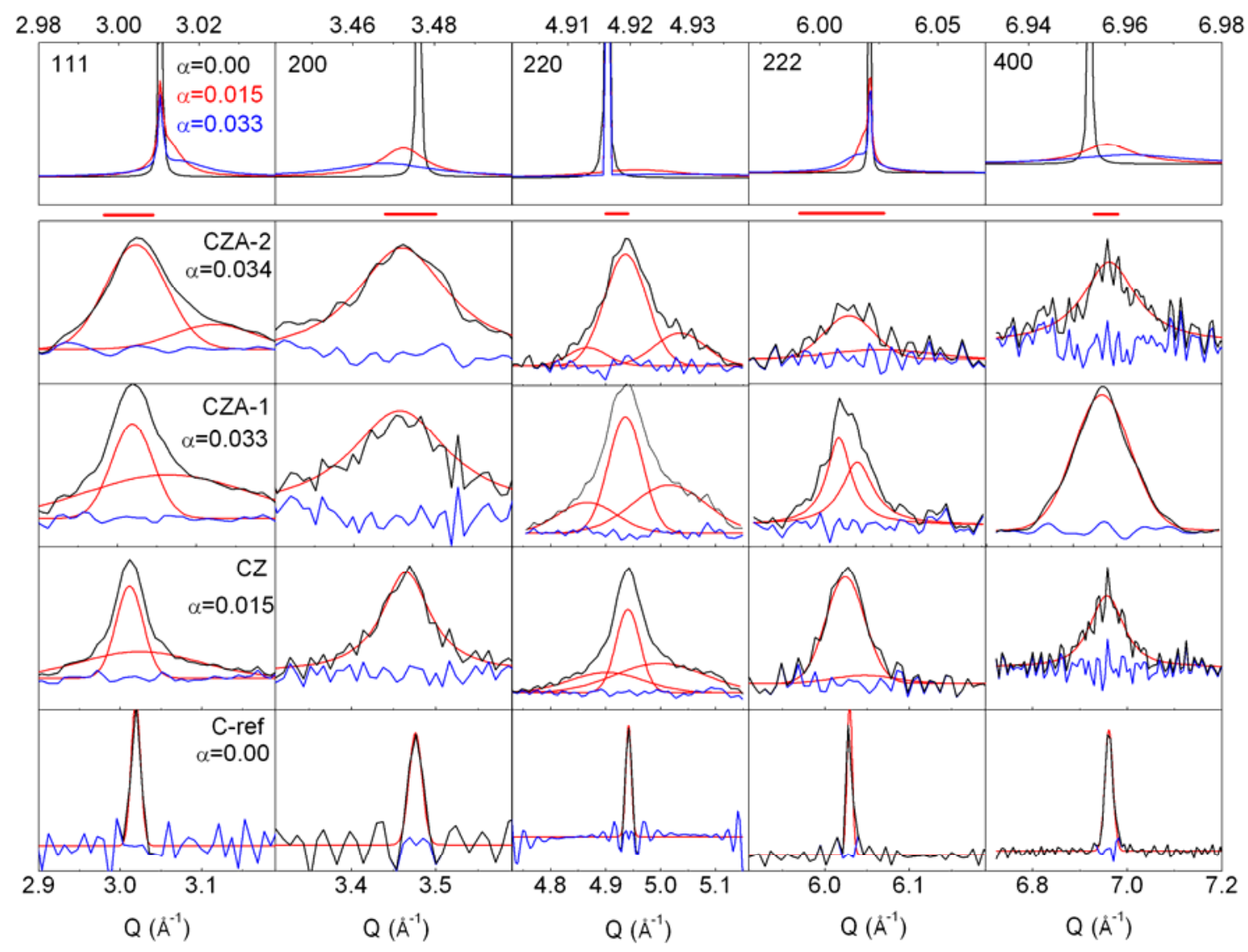

Figure 8: Deconvoluted smoothed peak profiles of CZA-2 ( $2^{\text {nd }}$ row), CZA-1 ( ${ }^{\text {rd }}$ row), CZ ( $4^{\text {th }}$ row) and C-ref (bottom row) compared to DIFFaX simulations of stacking fault-affected fcc-Cu ( $1^{\text {st }}$ row $)$. The profiles of the $111\left(1^{\text {st }}\right.$ column $), 200\left(2^{\text {nd }}\right.$ column $), 220$ ( $3^{\text {rd }}$ column$), 222\left(4^{\text {th }}\right.$ column) and 400 (last column) are shown. The same deconvolution procedure was applied to all peaks and all experimental patterns. Note the smaller Q-scale for the $1^{\text {st }}$ row on the uppermost panels, which is compared to the scale of row $2-5$ by the red lines between the $1^{\text {st }}$ and $2^{\text {nd }}$ row.

To analyze the experimental peak shapes, the raw data has been deconvoluted by the instrumental contribution (see supporting information for details). Although the signal-to-noise ratio of the neutron diffraction patterns of the catalysts is lower than recommended for a thorough peak shape analysis [42], the quality of the data after smoothing is sufficient for an estimation of the number and relative position of the different contributions to the total peak profile. It should be noted, that only smoothing-routines, which do not affect the peak position should be applied (e.g. weighted average or Savitzky-Golay, the latter was used here), As seen in Figure 8 , there is a good qualitative agreement between the simulated and measured peaks concerning the expected presence or absence of shoulders as a result of those peak components, which are affected by stacking faults. For example, a shoulder at the higher (lower) angle side of the 111 peak (222 peak) can be seen in all deconvoluted profiles of the catalyst samples, while the 200 peak (400 peak) do not show asymmetric broadening. Only negligible peak anisotropy was observed for the macrocrystalline C-ref sample. It is noted that, in contrast to the raw data, the deconvoluted peaks can be described by pure Gaussian, which indicates that macroscopic stresses can be neglected as a source of anisotropy. If only the affected components of the deconvoluted peaks are used for an estimation of the stacking fault probability $\alpha$ according to Eq.(8) generally higher values are obtained as reported in Figure 8, in particular for the CZA-1 catalyst.

Summarizing the results obtained until this point, it can be concluded that a consistent 
picture of the catalyst's microstructures emerges. There is a pronounced difference in particle and crystallite domain size between the binary $\mathrm{CZ}$ and the promoted CZA catalyst samples based on the results obtained by TEM and diffraction. Moreover, there are clear indications for the additional presence of structural imperfections that need to be taken into account for a complete description of the catalyst's microstructures. These indications are deviations from isotropic behavior observed in the Rietveld, Scherrer, and Wiliamson-Hall analyses and in the deconvoluted peak profiles. The individual deviations might not seem dramatic, but careful inspection clearly reveals a systematic pattern that can consistently be explained by the effect of stacking faults in the Cu nanoparticles. It is thus important to keep in mind that defects can have a significant effect on the quantitative results, e.g., of the Scherrer evaluation. It is noted that in addition to the effect of stacking faults discussed above in detail, also twin faulting, dislocations and microstain will affect the diffraction peak profiles. A disentanglement of these contributions is not straightforward and an attempt will be reported in the following section.

\subsection{Whole Powder Pattern Modeling (WPPM) including the effect of defects}

\subsubsection{WPPM of the neutron diffraction data}

The WPPM method is the most sophisticated way to extract microstructural information on defects from powder patterns. In this section, the comprehensive analysis of the catalyst's microstructure based on this method will be described. It is noted that accurate application of this method requires very high quality data in terms of signal-to-noise, preferably higher than in our neutron diffraction datasets. However, based on the previously obtained results from other methods that clearly confirm a visible effect of defects on the peak profiles and deliver reasonable starting parameters for the refinement and on the fact that the relatively high number of ten peaks can be simultaneously fitted, we report an attempt to use WPPM on the neutron diffraction pattern of the catalyst CZA-1 and the reference material C-ref, which are not affected by peak overlap due to the presence of other crystalline phases than $\mathrm{Cu}$.

WPPM includes a Fourier-synthesis from the parameter-dependent Fourier-coeffcients $\left(A_{\mathrm{L}}\right)$ that allow the refinement of a variety of defect parameters. In comparison with the manual pattern decomposition methods used for the Scherrer and Williamson-Hall analyses or the peak deconvolution method as shown above, the major advantage of this method is that, like in Rietveld fitting, the whole pattern information is used simultaneously. In contrast to the Rietveldalgorithm, the methodology behind WPPM is a convolutional fitting of the experimental pattern. Each contribution to a scattered intensity in an experimental powder pattern, whether due to the ideal structural model or originating from structural imperfections, can be described by a simplified Fourier-series of the type:

$$
I_{Q_{m}}=\int A_{L} \cdot \exp (2 \pi i L Q) d L
$$

In this study the program PM2K written by Leoni et al. [32] was used. In addition to the lattice parameter, also the domain size $D_{\text {ave }}$ and its statistical distribution, the stacking fault probability $\alpha$, the twinning probability $\beta$ (unfortunately usually given the same Greek letter like the integral peak breadth), the dislocation density $\rho$, the effective outer cut-off radius $R_{\mathrm{e}}$, the mixed average Burgers vector $b$ (describing magnitude and direction of a dislocation, due to edge and screw dislocations) and the stacking fault energy $\gamma$ are refined to model the peak profiles. Thus, the methodology simultaneously accounts for the effect of various types of defects and good starting parameters are essential to achieve a stable fit and physical meaningful results.

In a first step the contrast factors were refined with the pattern of C-ref without the inclusion of any defect model. The refined contrast factors are very similar to the used initial ones, calculated from literature values of the anisotropic elastic constants for the modified WilliamsonHall analysis (see supporting information) and applied to the catalyst sample. The crystal volume 
was calculated from the PSD determined by TEM (see above) and fixed during refinement. Initial values for $\alpha$ have been calculated according to Eq.(9) applied to the affected deconvoluted peaks (Figure 8 ) and those for $\beta$ were determined through the relation [38]:

$$
\beta=\frac{\alpha\left(\frac{1}{D_{200}}-\frac{1}{D_{111}}\right)}{1-\sqrt{\frac{3}{2}}}-1.5 \alpha
$$

using the Scherrer equation only on the unaffected deconvoluted peak contributions to determine $D_{\text {hkl }}$. The starting value for $D_{\text {ave }}$ was taken from the modified Williamson-Hall analysis. By using these initial parameters, a stable and reliable fit was achieved.

A direct comparison of the residuals from WPPM and Rietveld (Fig. 9) reveals the consistency of the underlying structural and defect model. It can be seen that the PM2K method deals much better with the experimental peak profiles and successfully models the anisotropic features that cause deviations in the difference curve of the Rietveld fit. For instance, comparison of the 331-420 region of the pattern reveals that the PM2K fit describes this doublet almost equally well as the model-free pattern decomposition method shown in Figure 4.

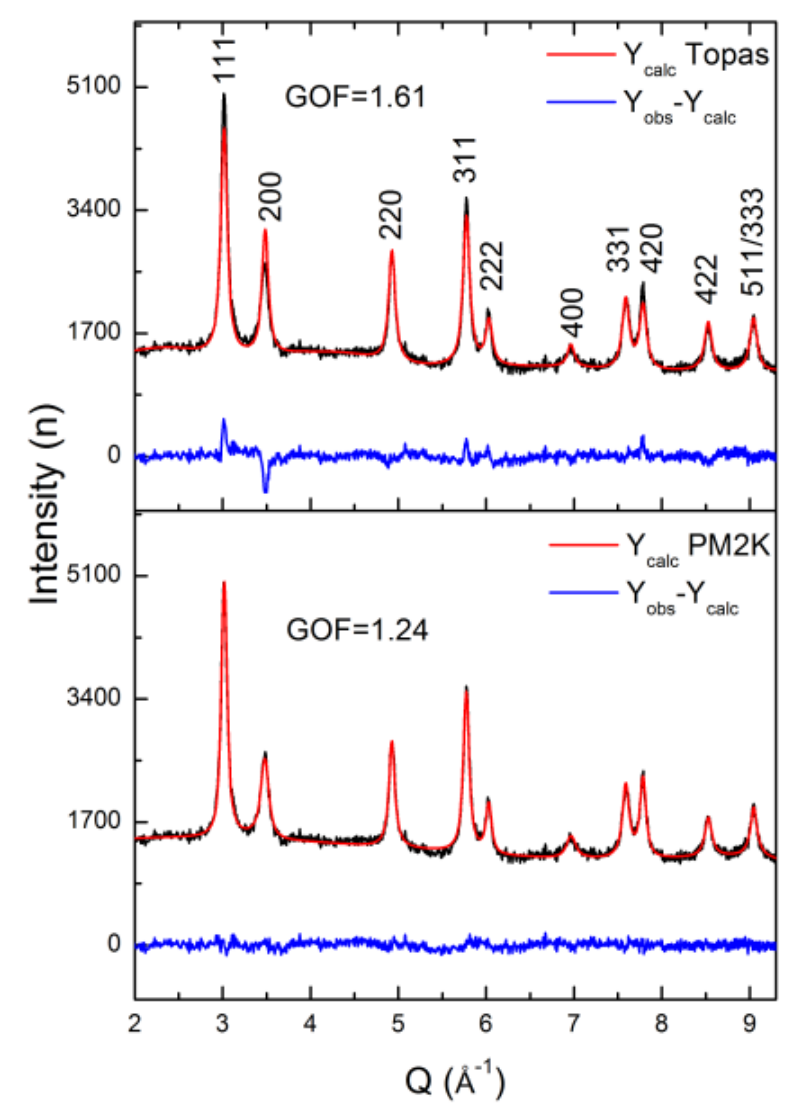

Figure 9: Comparison of the best fits using an ideal structure model (Rietveld, upper panel) and a methodology that accounts for the presence of lattice imperfections (WPPM, lower panel)

The results of the WPPM (Table 3) confirm once again that the difference between the macrocrystalline C-ref and the catalyst CZA-1 is not only the smaller domain (and particle) size of the latter, but that the $\mathrm{Cu}$ phase in the catalyst is substantially faulted and bears stacking faults, twin boundaries and dislocations that can explain the shifts and anisotropy of the peak profiles. The order of magnitude of the achieved stacking fault energy of the catalyst is in reasonable accordance with the literature results reported in the work of Reed and Schramm [56] who determined values in the range of 25 to $70 \mathrm{~mJ} / \mathrm{m}^{2}$ by XRD. Interestingly, the Burgers 
vector assuming an equal ratio between edge and screw dislocations, which is a general materials property of $\mathrm{Cu}$, is refined to a similar value in both materials despite their very different microstructure. The slight lattice contraction of the $\mathrm{Cu}$ nanoparticles in the catalyst sample that has already been observed by Rietveld refinement is confirmed by the PM2K results indicating an overall consistent fitting result.

Table 3: Refinement results of the PM2K-fit of the neutron diffraction patterns of C-ref and CZA-1 ( $a=$ lattice parameter; $D_{\text {ave }}$ : domain size; $\alpha=$ stacking fault probability; $\beta=$ the twinning probability; $\rho=$ dislocation density; $R_{\mathrm{e}}=$ effective outer cut-off radius; $b=$ the Burgers vector; $\gamma=$ stacking fault energy; GOF = goodness of fit).

\begin{tabular}{|l|l|l|l|l|l|l|l|l|l|}
\hline & $\begin{array}{l}\mathrm{a} \\
\left(10^{-1} \mathrm{~nm}\right)\end{array}$ & $\begin{array}{l}\mathrm{D}_{\text {ave }} \\
(\mathrm{nm})\end{array}$ & $\begin{array}{l}\alpha \\
\left(10^{-2}\right)\end{array}$ & $\begin{array}{l}\beta \\
\left(10^{-2}\right)\end{array}$ & $\begin{array}{l}\rho \\
\left(10^{-16} \mathrm{~m}^{-2}\right)\end{array}$ & $\begin{array}{l}\mathrm{R}_{\mathrm{e}} \\
\left(10^{-9} \mathrm{~m}\right)\end{array}$ & $\begin{array}{l}\mathrm{b} \\
\left(10^{-10} \mathrm{~m}\right)\end{array}$ & $\begin{array}{l}\gamma \\
\left(\mathrm{mJ} / \mathrm{m}^{2}\right)\end{array}$ & GOF \\
\hline C-ref & 3.6116 & $(21.13)$ & 0.006 & 0.16 & 0.14 & 0.0002 & 2.5538 & $(3.8)$ & 1.6779 \\
\hline CZA-1 & 3.6098 & 5.34 & 3.68 & 7.41 & 8.97 & 17.7 & 2.5525 & 18.1 & 1.2408 \\
\hline
\end{tabular}

Figure 10 shows an area-normalized comparison of the domain size distribution extracted from the WPPM-fit and the PSD as determined by particle counting in the TEM images. With the knowledge of the defective nature of the Cu particle, the discrepancy is easily explained by the difference in the underlying methodology. While in TEM the model is fitted to the appearance frequency of the particles (with a real space diameter), the WPPM distribution assumes a relatively narrow log-normal distribution around $D_{\text {ave }}$. Figure 10 can be interpreted in a way that each particle observed in TEM (approximately $10 \mathrm{~nm}$ ) roughly consists of two domains of a size of approximately $5 \mathrm{~nm}$, which are separated by a planar defect.

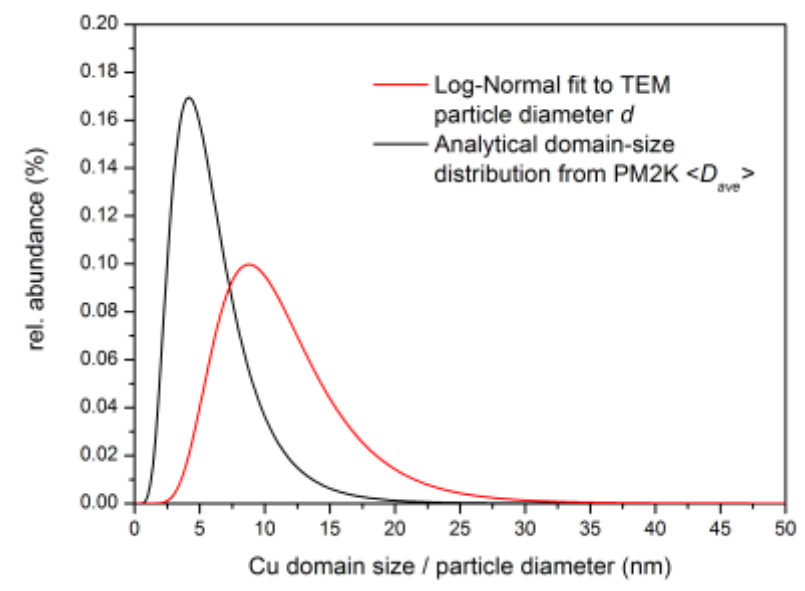

Figure 10: Comparison of area-normalized distribution functions for the domain sizes extracted from the WPPM and the particle sizes determined by TEM.

\subsubsection{Analysis based on standard-XRD}

The use of X-rays for diffraction experiments is much more common and as this is a laboratory technique also more convenient than neutron diffraction. In catalysis research XRD is a standard technique for catalyst characterization, but the application of microstructure analysis methods, with the exception of the Scherrer formula, is not routine. In this last section, we discuss how the features of anisotropy affect a standard XRD pattern for the example of the CZA-1 catalyst. The comparison between the Rietveld and WPPM fit is shown in Figure 11 for a selected section of the pattern.

The problems of the Rietveld fit are less obvious in the XRD data and only clearly seen for the first two reflections. However, if carefully inspected, Figure 11 shows similar phenomena as 
discussed previously in detail for the neutron data. The ideal structural model cannot simultaneously fit the more narrow width of the 111 and the shifted position of the 200 in an adequate manner. In the light of the above considerations, the experienced XRD scientist can find indications for defects in the typical down-up-down and up-down pattern of the difference curves of the 111 and 200 peaks even in this routine XRD pattern of standard quality. Indeed, the WPPM-fit can describe the experimental data much better taking the presence of defects into account. It is noted that also a full quantitative defect analysis of fcc metal nanoparticles is possible based using the WPPM method on XRD data as has been shown by the group of Weidenthaler for $\mathrm{Au} / \mathrm{ZrO}_{2}$ catalysts [8], but such complete analysis requires extremely high quality data.

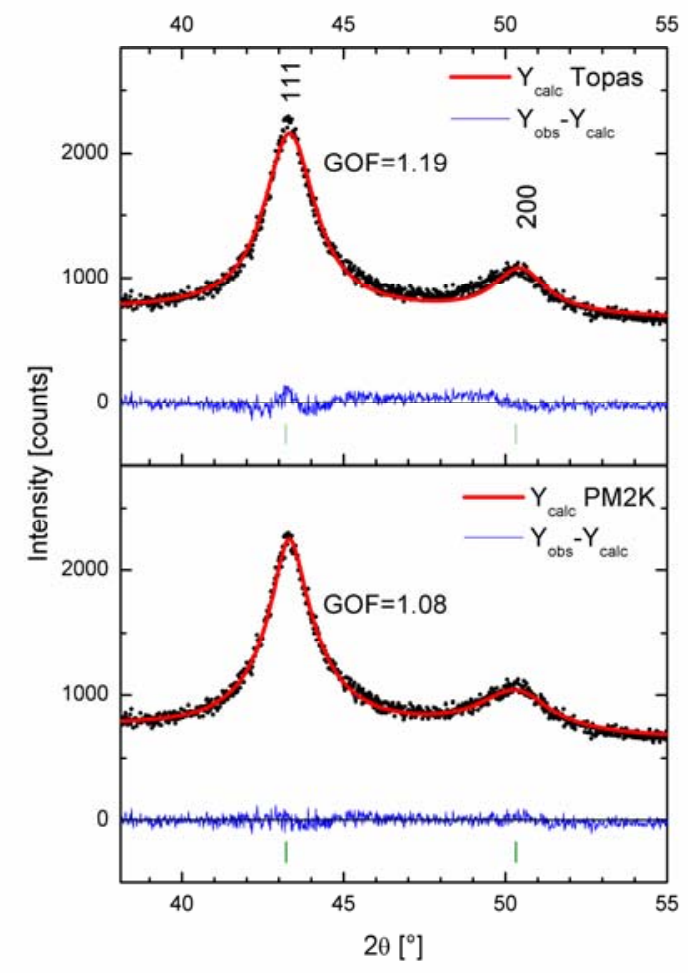

Figure 11: Section of the XRD pattern of the sample CZA-1 showing the region of the 111 and 200 peaks. The full patterns are shown as supporting information. The upper panel shows the best fit obtained by Rietveld refinement without considering structural imperfections, while the lower panel is the WPPM fit taking defects into account.

\section{Conclusion}

As discussed in the sections above, fcc metals like $\mathrm{Cu}$ can contain a large number of lattice imperfections of different dimensionalities ranging from vacancies over edge or screw type dislocations to areal defects such as stacking faults and twin boundaries. The observed intensity in a diffraction pattern is a convolution not only of the contributions due to the reflecting planes and instrumental broadening, but also of such lattice imperfections and of contributions due to size distribution. This can be used for a microstructural analysis of diffraction data, for which well-established methods are available whose application unfortunately still an exception in catalysis research. For the example of the methanol synthesis catalysts, it was shown that the presence of defects leads to deviations from an isotropic evolution of the peak profiles with $2 \theta$ in neutron diffraction patterns of several nano-structured Cu/ZnO-based materials. In comparison with a macrocrystalline pure $\mathrm{Cu}$ reference sample, clear indications for the presence of stacking 
faults in the catalysts' Cu particles have been observed in the residual of the Rietveld fits, in the scattering of the peak widths extracted from pattern decomposition, in the profile analysis using peak deconvolution as well as in the whole power pattern modeling. The existence of this type of defect was furthermore directly proven by HRTEM observations. In comparison with the domain size determined by diffraction methods, the particle size found the TEM images was significantly larger, which also points to the presence of planar defects that may separate the nanoparticles into individual smaller domains.

The deconvolution of the instrument function from the experimental pattern is mandatory to quantitatively describe the faulting probability. By an accurate determination of the apparent crystallite size with the help of the symmetric analytical fit of the unbroadened peak contributions by the classical Scherrer or modified Williamson-Hall analysis, physically meaningful values can be achieved. However, as the classical models for determining domain sizes from diffraction data rely on the assumption of uniform and mono-modal particle size distributions, complementary TEM studies are mandatory. Furthermore, it is noted that the results obtained by peak deconvolution exhibit high sensitivity to smoothing if such process was applied on noisy data. The (semi-)quantification of microstructural parameters like size and defect concentration of the catalysts CZ, CZA-1 and CZA-2 yielded different absolute values depending on the method used. Concerning the size, a clear trend was found among the three catalysts showing that the unpromoted catalysts $\mathrm{CZ}$ was least-nanosized, while the two differenty prepared $\mathrm{Al}_{2} \mathrm{O}_{3^{-}}$ promoted catalysts exhibit similar crystallite sizes. Depending on the method used, only slight but significant relative differences concerning these two samples were also observed. The large differences in the exposed $\mathrm{Cu}$ surface area between these catalysts must, however, be explained by embedding of the $\mathrm{Cu}$ particles in CZA-1 in the amorphous oxide matrix. Concerning the defect concentration, the relative results are not so clear. Based on the determination of the stacking fault probability from the peak shifts, CZ and CZA-1 show lower values than CZA-2 in agreement with the intrinsic catalytic activities. Based on the analysis of the deconvoluted peak profiles, both ternary CZA catalysts are richer in defects than the binary CZ. For the datasets reported in this study, we regard the peak shift method as more reliable regarding the relative differences of the catalysts, because the peak maxima can be more accurately determined even for unsmoothed profiles. In any case, care has to be taken when comparing absolute data of size or defect concentrations that have been determined by different methods. Concerning size data, Table 4 gives a summary of the methods that have been used in this work.

Table 4: Comparison of the different methods for size measurements

\begin{tabular}{|l|l|l|}
\hline Method & Result & Abbreviation \\
\hline Integral breadths & Column lengths in [hkl] & L \\
\hline Scherrer Formula & Apparent crystallite size in [hkl] & $D_{\text {eff }}$ \\
\hline Rietveld & Volume-weighted domain size & $D_{\text {vol }}$ \\
\hline TEM & Projected particle diameter & d \\
\hline Modified Williamson-Hall plot & Average domain size & $D_{\text {ave }}$ \\
\hline Whole powder pattern modelling & Average domain size & $D_{\text {ave }}$ \\
\hline
\end{tabular}

The most sophisticated method of defect analysis from powder diffraction data is the WPPM, but high quality data and proper initial parameters concerning instrumental contribution, faulting probability, dislocation density, linear expansion properties and particle size distribution are required, which is often a problem for realistic catalysts. However, a good measure for the quality of a fit is rather a matching, flat residual than numerical correlation factors. If this is obtained with physically plausible parameters and in general accordance with the results achieved with complementary methods, a reliable (semi-)quantitative microstructural characterization should also be possible for many catalysts. Qualitatively, the typical signature of planar defects on the peak profiles can be sometimes already seen in standard XRD evaluation, if the fitting residual is carefully inspected as was shown for the methanol synthesis catalysts 
under study. For such complex composite nano-materials, we recommend the comparative application of different line profile analysis methods to scan for consistent anisotropic effects by looking at more than one reflection. This can already by easily done using a simple pattern decomposition method like the Williamson-Hall analysis. 


\section{References}

1. Dahl S, Logadottir A, Egeberg RC, Larsen JH, Chorkendorff I, Tornqvist E, Norskov JK (1999) Role of steps in N-2 activation on Ru(0001). Phys Rev Lett 83 (9):1814-1817. doi:DOI 10.1103/PhysRevLett.83.1814

2. Rostrup-Nielsen J, Norskov JK (2006) Step sites in syngas catalysis. Topics in Catalysis 40 (1-4):45-48. doi:DOI 10.1007/s11244-006-0107-5

3. Abild-Pedersen F, Greeley J, Norskov JK (2005) Understanding the effect of steps, strain, poisons, and alloying: Methane activation on Ni surfaces. Catalysis Letters 105 (1-2):9-13. doi:DOI 10.1007/s10562-005-7998-9

4. Jiang T, Mowbray DJ, Dobrin S, Falsig H, Hvolbaek B, Bligaard T, Norskov JK (2009) Trends in CO Oxidation Rates for Metal Nanoparticles and Close-Packed, Stepped, and Kinked Surfaces. J Phys Chem C 113 (24):10548-10553. doi:Doi 10.1021/Jp811185g

5. Freund HJ (2010) Model Studies in Heterogeneous Catalysis. Chem-Eur J 16 (31):93849397. doi:DOI 10.1002/chem.201001724

6. Datye AK (2003) Electron microscopy of catalysts: recent achievements and future prospects. Journal of Catalysis 216 (1-2):144-154. doi:Doi 10.1016/S0021-9517(02)00113-6

7. Weidenthaler $C$ (2011) Pitfalls in the characterization of nanoporous and nanosized materials. Nanoscale 3 (3):792-810. doi:Doi 10.1039/COnr00561d

8. Pandey AD, Guttel R, Leoni M, Schuth F, Weidenthaler C (2010) Influence of the Microstructure of Gold-Zirconia Yolk-Shell Catalysts on the CO Oxidation Activity. J Phys Chem C 114 (45):19386-19394. doi:Doi 10.1021/Jp106436h

9. Vogel W (1990) Size Distributions of Supported Metal-Catalysts - an Analytical X-Ray-Line Profile Fitting Routine. Journal of Catalysis 121 (2):356-363. doi:Doi 10.1016/00219517(90)90244-E

10. Tsybulya SV, Kryukova GN, Goncharova SN, Shmakov AN, Balzhinimaev BS (1995) Study of the Real Structure of Silver Supported Catalysts of Different Dispersity. Journal of Catalysis 154 (2):194-200. doi:DOI 10.1006/jcat.1995.1160

11. Bautista FM, Campelo JM, Garcia A, Luna D, Marinas JM, Quiros RA, Romero AA (1998) Influence of surface support properties on the liquid-phase selective hydrogenation of phenylacetylene on supported nickel catalysts. Catalysis Letters 52 (3-4):205-213. doi:Doi 10.1023/A:1019008528391

12. Diffraction Analysis of the Microstrucutre of Materials (2004). Springer Series in Materials Science,

13. Mittemeijer EJ, Welzel U (2008) The state of the art of the diffraction analysis of crystallite size and lattice strain. Z Kristallogr 223:552-560

14. Savaloni H, Gholipour-Shahraki M, Player MA (2006) A comparison of different methods for $\mathrm{x}$-ray diffraction line broadening analysis of $\mathrm{Ti}$ and $\mathrm{Ag}$ UHV deposited thin films: nanostructural dependence on substrate temperature and film thickness. Journal of Physics D: Applied Physics 39 (10):2231

15. Behrens M, Schlögl R (2012) X-Ray Diffraction and Small Angle X-Ray Scattering. In: Characterization of Solid Materials and Heterogeneous Catalysts. Wiley-VCH Verlag GmbH \& Co. KGaA, pp 609-653. doi:10.1002/9783527645329.ch15

16. Kasatkin I, Kurr P, Kniep B, Trunschke A, Schlogl R (2007) Role of lattice strain and defects in copper particles on the activity of $\mathrm{Cu} / \mathrm{ZnO} / \mathrm{Al} 2 \mathrm{O} 3$ catalysts for methanol synthesis. Angew Chem Int Edit 46 (38):7324-7327. doi:DOI 10.1002/anie.200702600

17. Gunter MM, Ressler T, Bems B, Buscher C, Genger T, Hinrichsen O, Muhler M, Schlögl R (2001) Implication of the microstructure of binary $\mathrm{Cu} / \mathrm{ZnO}$ catalysts for their catalytic activity in methanol synthesis. Catalysis Letters 71 (1-2):37-44

18. Behrens M, Studt F, Kasatkin I, Kühl S, Hävecker M, Abild-Pedersen F, Zander S, Girgsdies F, Kurr P, Kniep B-L, Tovar M, Fischer RW, Norskov JK, Schlögl R (2012) The Active Site of Methanol Synthesis over Cu/ZnO/Al2O3 Industrial Catalysts. Science 336 (6083):893-897

19. Behrens $\mathrm{M}$ (2009) Meso- and nano-structuring of industrial $\mathrm{Cu} / \mathrm{ZnO} /(\mathrm{Al} 2 \mathrm{O} 3)$ catalysts. Journal of Catalysis 267 (1):24-29. doi:10.1016/j.jcat.2009.07.009

20. Waller D, Stirling D, Stone FS, Spencer MS (1989) Copper-zinc oxide catalysts. Activity in relation to precursor structure and morphology. Faraday Discussions of the Chemical Society $87: 107-120$

21. Spencer MS (1999) The role of zinc oxide in Cu/ZnO catalysts for methanol synthesis and the water-gas shift reaction. Topics in Catalysis 8:259-266 
22. Behrens M, Furche A, Kasatkin I, Trunschke A, Busser W, Muhler M, Kniep B, Fischer R, Schlogl R (2010) The Potential of Microstructural Optimization in Metal/Oxide Catalysts: Higher Intrinsic Activity of Copper by Partial Embedding of Copper Nanoparticles. Chemcatchem 2 (7):816-818. doi:10.1002/cctc.201000017

23. Klug HP, Alexander LE (1974) X-Ray Diffraction Procedures. John Wiley \&amp; Sons, New York,

24. Balzar D, Audebrand N, Daymond MR, Fitch A, Hewat A, Langford JI, Le Bail A, Louër D, Masson O, McCowan CN, Popa NC, Stephens PW, Toby BH (2004) Size--strain linebroadening analysis of the ceria round-robin sample. J Appl Crystallogr 37 (6):911-924

25. Többens DM, Tovar M (2002) Peak shape at the axially focusing E9 powder diffractometer theoretical and experimental description. Applied Physics A: Materials Science \&amp; Processing 74:s136-s138

26. Caglioti G, Paoletti A, Ricci FP (1958) Choice of collimators for a crystal spectrometer for neutron diffraction. Nuclear Instruments 3 (4):223-228

27. Rietveld HM (1969) A profile refinement method for nuclear and magnetic structures. Journal of Applied Crystallography 2 (2):65-71

28. Wertheim GK, Butler MA, West KW, Buchanan DNE (1974) Determination of the Gaussian and Lorentzian content of experimental line shapes. Review of Scientific Instruments 45 (11):1369-1371

29. Cheary RW, Coelho AA, Cline JP (2004) Fundamental Parameters Line Profile Fitting in Laboratory Diffractometers. J Res Natl Inst Stand Technol 109:1-25

30. Le Bail A, Duroy H, Fourquet JL (1988) Ab initio structure determination of LiSbWO \$_6 by X-ray powder diffraction. Materials Research Bulletin 23 (3):447-452

31. Treacy MMJ, Newsam JM, Deem MW (1991) A General Recursion Method for Calculating Diffracted Intensities from Crystals Containing Planar Faults. Proceedings of the Royal Society of London Series A: Mathematical and Physical Sciences 433 (1889):499-520

32. Leoni M, Confente T, Scardi P (2006) PM2K: a flexible program implementing Whole Powder Pattern Modelling. Z Kristallogr Suppl 2006 (23):249-254

33. Twigg MV, Spencer MS (2003) Deactivation of copper metal catalysts for methanol decomposition, methanol steam reforming and methanol synthesis. Top Catal 22 (3-4):191203

34. Romeo M, Da Costa V, Bardou F (2003) Broad distribution effects in sums of lognormal random variables. The European Physical Journal B - Condensed Matter and Complex Systems 32:513-525

35. Gai PL, Boyes ED (2003) Electron Microscopy in Heterogeneous Catalysis. Institute of Physics Publishing,

36. Yao MH, Smith DJ, Datye AK (1993) Comparative-Study of Supported Catalyst Particles by Electron-Microscopy Methods. Ultramicroscopy 52 (3-4):282-288. doi:Doi 10.1016/03043991(93)90037-X

37. Delhez R, Keijser TH, Mittemeijer EJ (1982) Determination of crystallite size and lattice distortions through X-ray diffraction line profile analysis. Fresenius' Journal of Analytical Chemistry $312: 1-16$

38. Warren BE (1990) X-Ray Diffraction. Dover Publications,

39. Balogh L, Ribárik G, Ungár T (2006) Stacking faults and twin boundaries in fcc crystals determined by x-ray diffraction profile anaylsis. J Appl Phys 100:023512-023512

40. Scherrer P (1918). Göttinger Nachrichten Gesell 2:98-98

41. Patterson AL (1939) The Scherrer Formula for X-Ray Particle Size Determination. Phys Rev 56:978-982

42. Langford JI, Louër D (1996) Powder Diffraction. Rep Prog Phys 59:131-234

43. Langford JI, Wilson AJC (1978) Scherrer after sixty years: A survey and some new results in the determination of crystallite size. J Appl Crystallogr 11 (2):102-113

44. Williamson GK, Hall WH (1953) X-Ray Line Broadening from Filed Aluminium and Wolfram. Acta Metall 1:22-31

45. Brandstetter S (2008) Williamson-Hall anisotropy in nanocrystalline metals: X-ray diffraction experiments and atomistic simulations. Acta Mater 56:165-176

46. Ungar T, Tichy G (1999) The Effect of Dislocation Contrast on X-Ray Line Profiles in Untextured Polycrystals. Phys Stat Sol A 171:425-425

47. Warren BE (1961) X-ray Measurements of Stacking Fault Widths in fcc Metals. J Appl Phys $32: 2428-2428$ 
48. Ungar T, Borbely A (1996) The effect of dislocation contrast on xray line broadening: A new approach to line profile analysis. Appl Phys Lett 69:3173-3173

49. Gubicza J, Szepvolgi J, Mohai I, Zsoldos L, Ungar T (2000) Particle size distribution and dislocation density determined by high resolution X-ray diffraction in nanocrystalline silicon nitride powders. Mater Sci Eng A 280:263-269

50. Ungar T, Gubicza J, Ribarik G, Borbely A (2001) Crystallite size distribution and dislocation strucutre determined by diffraction profile analysis: principles and practical applications to cubic and hexagonal crystals. J Appl Cryst 34:298-310

51. Defect and Microstructure Analysis by Diffraction (2000). OUP//International Union of Crystallography,

52. Warren BE (1959) X-ray studies of deformed metals. Progress in Metal Physics 8 (0):147202

53. Paterson MS (1952) X-Ray Diffraction by Face-Centered Cubic Crystals with Deformation Faults. J Appl Phys 23 (8):805-811

54. Kandemir T (2013) In-situ study of catalytic processes at high temperatures: Neutron diffraction of $<p>a$ methanol synthesis catalyst under industrial conditions. Angew Chem-Int Ed DOI: 10.1002/anie.201209539

55. Velterop L, Delhez R, de Keijser TH, Mittemeijer EJ, Reefman D (2000) X-ray diffraction analysis of stacking and twin faults in f.c.c. metals: a revision and allowance for texture and non-uniform fault probabilities. J Appl Crystallogr 33 (2):296-306

56. Reed RP, Schramm RE (1974) Relationship between stacking-fault energy and x-ray measurements of stacking-fault probability and microstrain. Journal of Applied Physics 45 (11):4705-4711 